QUARTERLY OF APPLIED MATHEMATICS

VOLUME LXIX, NUMBER 1

MARCH 2011, PAGES 123-146

S 0033-569X(2010)01236-7

Article electronically published on December 29, 2010

\title{
EXISTENCE OF TRAVELING WAVES IN A SIMPLE ISOTHERMAL CHEMICAL SYSTEM WITH THE SAME ORDER FOR AUTOCATALYSIS AND DECAY
}

\author{
BY \\ JE-CHIANG TSAI \\ Department of Mathematics, National Chung Cheng University, 168, University Road, Min-Hsiung, \\ Chia-Yi 621, Taiwan
}

\begin{abstract}
The reaction-diffusion systems which are based on an isothermal autocatalytic chemical reaction involving both an autocatalytic step of the $(m+1)$ th order $(A+m B \rightarrow(m+1) B)$ and a decay step of the same order $(B \rightarrow C)$ have very rich and interesting dynamics. Previous studies in the literature indicate that traveling waves play a key role in understanding these interesting dynamical phenomena. However, there is a lack of rigorous proof of the existence of traveling waves to this system. Here we generalize this isothermal autocatalytic chemical reaction model and provide a rigorous proof of the existence of traveling waves for the resulting reaction-diffusion system which also includes the systems arising from epidemiology and the microbial growth in a flow reactor.
\end{abstract}

1. Introduction. Since the pioneering works of Fisher [9] and of Kolmogorov, Petrovsky and Piskunov [23, traveling waves in a wide array of biological and chemical systems, based on the interaction between reaction and diffusion processes, have been extensively investigated. In many realistic systems, traveling waves can be initiated by a highly localized disturbance of a stable rest state (e.g., a highly localized increase in ion concentrations). A typical example is the Hodgkin-Huxley model [13] which describes the electrical activity across membranes of never cells. It is known that if a short, but sufficiently large, burst of simulating current is injected into a nerve axon, a so-called action potential wave can then be generated. In this paper, we will discuss a system which enjoys a similar phenomenon. Specifically, this system is governed by the following equations:

$$
u_{t}=d_{1} u_{x x}-f(u) v^{m}, \quad v_{t}=d_{2} v_{x x}+[f(u)-K] v^{m},
$$

Received July 29, 2009.

2000 Mathematics Subject Classification. Primary 34A34, 34A12, 35K57.

Key words and phrases. Isothermal autocatalytic chemical reaction, traveling waves, reaction-diffusion systems, centre manifold.

The author is supported in part by the National Science Council of Taiwan.

E-mail address: tsaijc@math.ccu.edu.tw 
where $d_{1}>0, d_{2}>0, K \geq 0$, and $m \geq 1$ are constants, and $f(u)$ is a smooth function to be specified later. The system (1.1) has been proposed to describe wave propagation in a simple isothermal chemical reaction model with the same order for autocatalysis and decay. It has also been considered as a simple model for the spread of infectious diseases (see Kermack and McKendric [22] and Bailey [3]) and introduced to discuss a microbial growth for the nutrient in a flow reactor (see Kennedy and Aris 21] and Ballyk et al. 44). We will detail them below.

We are first concerned with the isothermal autocatalytic chemical reaction. Generally speaking, chemical reacting systems always involve many elementary reaction steps. As a first step to understand the effect of these elementary reaction steps on the chemical reacting systems, we assume that only the reactant $A$ and the autocatalyst $B$ are involved in the overall reaction, and the reaction scheme is represented by

$$
A+m B \rightarrow(m+1) B \quad \text { with rate } k_{1} u v^{m} \quad \text { (autocatalytic step). }
$$

Here $u$ and $v$ denote the concentration of the reactant $A$ and autocatalyst $B$, respectively, the $k_{1}$ is the rate constant of the autocatalysis, and $m$ is the order of the autocatalysis. In general, the reaction order $m$ in realistic models is determined empirically, and so $m$ is not necessarily an integer (see Kay et al. 20]). Here we require that $m \geq 1$. Mathematically, we can apply the mass-action law to (1.2) to obtain the system (1.1) with $f(u)=k_{1} u$ and $K=0$. The cubic autocatalytic reaction (the autocatalytic step (1.2) with $m=2$ ) has been successfully applied to model many realistic systems, for example, the iodate-arsenous acid reaction in the arsenous acid excess case (see Saul and Showalter [31]), and the hydroxylamine-nitrate reaction proposed by Gowland and Stedman [10. Moreover, the cubic autocatalytic reaction forms a fundamental step in the almost isothermal flames in the carbon-sulphide-oxygen reaction presented by Voronkov and Semenov 33. Experimental evidence shows that wavefront propagation can be observed in the chemical system for which the autocatalytic step (1.2) with $m=1$ or 2 forms a fundamental step (see Zaikin and Zhabotinskii 34]). Precisely, if the reactant $A$ initially distributes uniformly in the whole space, and a quantity of the autocatalyst, $B$, is added locally into this system, then $A$ and $B$ will react in this initial zone. This will lead to the concentration gradients, and then the advancing wave front of the reactant $A$ (or the autocatalyst $B$ ) is thus generated and will propagate out from this initial zone. Mathematically, Billingham, Merkin, and Needham [25, 5, 6, 28] have applied the numerical and asymptotical analysis to the system (1.1) with $f(u)=k_{1} u$ and $K=0$ to confirm such a phenomenon of wave front propagation. We note that the rigorous proof of the existence of traveling wave front solutions to the system (1.1) with $f(u)=k_{1} u$ and $K=0$ is given by Qi [30] and Chen and Qi [8] (see also Marion 24] and Ai and Huang 2 for the general reaction term $f$ ).

On the other hand, the analysis by Billingham, Merkin, and Needham [25, 5, 6, 28] shows that no matter how small the amount of the autocatalyst $B$ is introduced locally into the system (1.1) with $f(u)=k_{1} u$ and $K=0$, traveling waves are always generated. This particular feature seems to be contrary to the fact that in many chemical systems, the initial input of an autocatalyst into the system must be above a threshold concentration for the initialization of traveling waves. To overcome this controversy on the 
absence of the threshold concentration in the system (1.1) with $f(u)=k_{1} u$ and $K=0$, Gray [11] has suggested that the autocatalyst cannot be indefinitely stable; instead, it should undergo subsequent change. Along this line, Merkin and Needham [26, 27, 29] have assumed that the autocatalyst $B$ decays to the inert product $C$ at a rate of order $m$,

$$
B \rightarrow C \quad \text { with rate } k_{2} v^{m} \quad \text { (decay step). }
$$

Then by applying the mass-action law to (1.2)-(1.3), it yields the system (1.1) with $f(u)=k_{1} u$ and $K=k_{2}>0$. This modified version of the system (1.1) has been analyzed by Merkin and Needham 26, 27, 29, and their asymptotical analysis indicates the existence of the threshold concentration of the autocatalyst $B$ for the generation of traveling waves. We remark that unlike the case with $f(u)=k_{1} u$ and $K=0$, the wave profile for the reactant $A$ is of front type and the one for the autocatalyst $B$ is of pulse type due to the decay. Therefore the system (1.1) with $f(u)=k_{1} u$ and $K=k_{2}>0$ seems to be more consistent with experimental observations (see Zaikin and Zhabotinskii 34]). For more detail about this model, we refer the reader to Kay et al. [20].

Next, we note that the system (1.1) can arise in the context of the spread of infectious diseases. Specifically, if $m=1$ and $f(u)=\beta u$, then the system (1.1) is a classical diffusive epidemic model of Kermack-McKendric [22, 3], which describes the interaction between the susceptible individual $u$ and the infected individual $v$. Here positive constants $\beta$ and $K$ denote the contact rate and the removal rate, respectively. Note that if the removal rate $K=0$, it would imply that the infected species would remain indefinitely infective. Hence it is reasonable to assume that the removal rate $K$ is positive in this case. Finally, the system (1.1) with $m=1$ and $f(u)=m_{1} u /\left(m_{2}+u\right)$ has been introduced by Ballyk et al. [4] (see also Kennedy and Aris 21]) as a model to explore the effects of the motility on the ability of a bacterial population to survive in a flow reactor and/or to be a good competitor for a limiting nutrient in a mixed culture. In this case, $u$ and $v$ denote the concentration of the nutrient and the density of the bacterial population, respectively, while $f(u)$ is the nutrient uptake rate at nutrient concentration $u$, and the parameter $K>0$ is the cell death rate.

From the above discussion, we see that a good knowledge of traveling waves to the system (1.1) may provide great insight to the dynamical behavior of solutions to the system (1.1). We also note that there is a lack of rigorous proof of the existence of traveling waves to the system (1.1) with $f(u)=k_{1} u, K>0$ and $m>1$ corresponding to the reaction scheme (1.2)-(1.3). Motivated by these two reasons and the rich applications of the system (1.1), the aim of this paper is to explore the existence of traveling wave solutions of (1.1) with $K>0$ and $m>1$. To begin with, we give the exact definition of a traveling wave solution to the system (1.1). A set of nonnegative functions $(U, V) \in$ $C^{2}(\mathbb{R}) \times C^{2}(\mathbb{R})$ is a traveling wave solution of (1.1) if for some $c \in \mathbb{R},(u(x, t), v(x, t))=$ $(U(z), V(z))$ with $z:=x-c t$ is a solution of (1.1) and $(U(z), V(z))$ satisfies the additional conditions $\lim _{z \rightarrow-\infty}(U, V)=\left(u_{0}, 0\right)$ and $\lim _{z \rightarrow+\infty}(U, V)=\left(u^{0}, 0\right)$ for some $u_{0} \in\left(0, u_{K}\right)$ and $u^{0} \in\left(u_{K}, \infty\right)$. Here $u_{K}$ is a positive constant satisfying $f\left(u_{K}\right)=K$, and $c$ is the wave speed. With a simple calculation, it follows that $(U, V)$ satisfies the ordinary 
differential system

$$
d_{1} U^{\prime \prime}+c U^{\prime}-f(U) V^{m}=0, \quad d_{2} V^{\prime \prime}+c V^{\prime}+[f(U)-K] V^{m}=0
$$

subject to the boundary conditions

$$
\lim _{z \rightarrow-\infty}\left(U, U^{\prime}, V, V^{\prime}\right)=\left(u_{0}, 0,0,0\right), \quad \lim _{z \rightarrow+\infty}\left(U, U^{\prime}, V, V^{\prime}\right)=\left(u^{0}, 0,0,0\right),
$$

where the prime denotes $d / d z$.

We remark that in the real physical situation, as for the boundary values, $u^{0}$ can only be specified, and $u_{0}$ is previously unknown and should be determined. The existence of traveling wave solutions of (1.1) for some special choices of functions $f$ and $K>0$ has been studied by numerous authors. When $d_{1}=0$ and $m=1$, Kennedy and Aris [21] and Källén [19] have solved the problem (1.4)-(1.5) for the special cases $f(u)=u$ and $f(u)=m_{1} u^{m_{2}} /\left(m_{3}+u^{m_{2}}\right)$, respectively, where $m_{i}, i=1,2,3$, is a positive constant. For the other extreme case $d_{1}>0, d_{2}=0$ and $m=1$, Hosono and Ilyas [14] have treated the problem (1.4)-(1.5) for the particular choice $f(u)=u$. When the diffusion coefficients $d_{1}$ and $d_{2}$ are positive and $m=1$, Smith and Zhao 32 have addressed the problem (1.4)-(1.5) where $f(u)=m_{1} u /\left(m_{2}+u\right)$ for some positive constants $m_{1}$ and $m_{2}$, and $d_{1}$ is sufficiently small, and Hosono and Ilyas [15] have considered the case $f(u)=u$ with any positive constants $d_{1}$ and $d_{2}$. We remark that the methods of the above-mentioned results strongly depend on the particular choice of $f$ and $m=1$, and so cannot be applied to the general class of functions $f$ and $m>1$. In particular, these methods cannot work for the case: $f(u)=k_{1} u$ and $m>1$ which corresponds to the isothermal autocatalytic chemical reaction (1.2)-(1.3). Recently, Huang [17] (see also Ai and Huang [1]) has developed a method to deal with the problem (1.4)-(1.5) with arbitrary positive constants $d_{1}$ and $d_{2}$ for a general class of functions $f$ which include all the reaction terms $f$ mentioned above. However, the parameter $m$ in Huang [17] is restricted to $m=1$.

Now we turn to the case $m>1$. Indeed, Hosono 16 has shown the existence of traveling wave solutions of the system (1.1) with $d_{1}=0$ and the function $f$ restricted to the form $f(u)=k_{1} u$ which corresponds to the higher-order isothermal autocatalytic chemical reaction (1.2)-(1.3) with immobile reactant. More recently, Guo and Tsai 12 have considered the other extreme case: $d_{1}>0, d_{2}=0, m>1$ and a general class of functions $f$. Moreover, the result in Guo and Tsai [12] can be applied to show the existence of traveling wave solutions of higher-order isothermal autocatalytic chemical reactions (1.2)-(1.3) with immobile autocatalyst. On the other hand, since the methods in Hosono [16] and Guo and Tsai [12] are restricted to either $d_{1}=0$ or $d_{2}=0$, and hence, cannot be used to show the more interesting case: $d_{1}>0$ and $d_{2}>0$. In this paper, we shall study the problem (1.4)-(1.5) for the case where $d_{1}>0, d_{2}>0, m>1$, and $f$ satisfies the same assumptions as in Huang [17] (see (A1)-(A3) below). More precisely, $f$ satisfies the following conditions:

(A1) $f:[0, \infty) \rightarrow \mathbb{R}$ is differentiable and $f(0)=0$.

(A2) There exists a unique number $u_{K}>0$ such that $f\left(u_{K}\right)=K$ and

$$
f(u) \in(0, K) \text { for all } u \in\left(0, u_{K}\right), f(u)>K \text { for all } u \in\left(u_{K}, \infty\right) .
$$


(A3) $f$ is increasing on $\left[0, \xi^{*}\right]$ for some $\xi^{*} \in\left(0, u_{K}\right)$ and satisfies

$$
\int_{0^{+}}^{u_{K}} \frac{1}{f(u)} d u=+\infty
$$

The assumption (A3) simply implies that for $u$ close to 0 , the function $f(u)$ approximates the power function $\alpha u^{n}$ for some positive number $\alpha$ and positive number $n \geq 1$. Typical examples for $\left(f, u_{K}, K\right)$ satisfying the assumptions (A1)-(A3) are $\left(f(u), u_{K}, K\right)=(u, 1,1)$ and $\left(f(u), u_{K}, K\right)=\left(m_{1} u /\left(m_{2}+u\right), 1, m_{1} /\left(m_{2}+1\right)\right)$, where $m_{1}$ and $m_{2}$ are positive constants. Now we can state our main result on the existence of traveling waves of the system (1.1) in the following theorem.

Theorem 1.1. Let the constants $d_{1}, d_{2}$ and $K$ be positive, and $m>1$. Suppose that $f$ satisfies the assumptions (A1)-(A3). Then, for each given $u^{0}>u_{K}$, there is a positive constant $c\left(u^{0}\right)$ such that for each $c>c\left(u^{0}\right)$, there exists a unique solution $(u, v)$ (up to a translation) to (1.4)-(1.5) for some $u_{0} \in\left(0, u_{K}\right)$. Moreover, we have $u^{\prime}>0$ on $\mathbb{R}$, and $v^{\prime}>0$ on $\left(-\infty, z_{1}\right)$ and $v^{\prime}<0$ on $\left(z_{1},+\infty\right)$ for some $z_{1} \in \mathbb{R}$.

We conclude the introduction with three remarks. The first remark is that the reaction terms $f(u)$ in the above-mentioned models $22,3,21,19,26,27,29,4$, are smooth and increasing in $u$, and $f(0)=0$ and $f^{\prime}(0)>0$. Hence $f$ in these models satisfies the assumptions (A1)-(A3). Therefore our result also shows the existence of traveling wave solutions to the system (1.1) with $f(u)=k_{1} u$ and $K=k_{2}$ which corresponds to an isothermal autocatalytic chemical reaction of order $m>1$ in which the autocatalyst is assumed to decay to the inert product at a rate of the same order $m$.

Next, the restriction on $c\left(u^{0}\right)$ is technical due to the argument employed in Lemma2.3. One may expect that there is a minimum wave speed. However, we are unable to prove this conjecture, which will be left to our future study. Concerning the uniqueness of the profiles of traveling waves, we note that given $u^{0}>u_{K}$ and $c>c\left(u^{0}\right)$, if $f$ is monotone increasing, then one may expect that there is a unique $u_{0} \in\left(0, u_{K}\right)$ such that a traveling wave solution $(U, V)$ connecting $\left(u_{0}, 0\right)$ and $\left(u^{0}, 0\right)$ exists. This has been confirmed for the case $m=1$ in Huang [18. However, the method in Huang [18] relies heavily on the fact that a traveling wave profile $(U(z), V(z))$ for the case $m=1$ lies on the unique unstable manifold for negative $z$ with $|z| \gg 1$. By contrast, a traveling wave profile $(U(z), V(z))$ for the case $m>1$ lies on the centre manifold for negative $z$ with $|z| \gg 1$. Hence it seems that this method might not be applied to the case $m>1$. The study of these open questions will be our future study.

The final remark concerns the method of our proof. Indeed, the method of our proof is based on the method in Huang [17, where the author only considers the case $m=1$. On the other hand, for the case $m>1$, the Liapunov functions constructed in Huang [17] might not enjoy good properties as in the case $m=1$, and so some additional estimates for the Liapunov functions constructed in Huang [17] are needed for the case $m>1$ (see the proofs of Lemmas 3.2 and 3.4 .

Finally, the plan of this paper is as follows. In Section 2, with the aid of the centre manifold theory, we establish the local existence and uniqueness of the solution $(U, V)$ of (1.4) subject to the condition $\lim _{z \rightarrow-\infty}\left(U(z), U^{\prime}(z), V(z), V^{\prime}(z)\right)=\left(u_{0}, 0,0,0\right)$ for a 
given $u_{0} \in\left(0, u_{K}\right)$. Then with the phase plane argument, such a local solution can be shown to exist globally provided that $c$ is sufficiently large. Finally, Section 3 is devoted to establish several auxiliary lemmas which form the basis for the proof of Theorem 1.1.

2. Existence of traveling wave solutions. For technical convenience, we will impose the extra assumption (A4) on $f$ :

$$
\text { (A4) } \liminf _{u \rightarrow \infty} f(u)>K \text {. }
$$

However, this assumption will be removed in the proof of Theorem 1.1

By employing the rescalings

$$
\tilde{x}=\frac{x}{\sqrt{d_{2}}}, \quad d=\frac{d_{1}}{d_{2}},
$$

the system (1.1) can be reduced to

$$
u_{t}=d u_{x x}-f(u) v^{m}, \quad v_{t}=v_{x x}+[f(u)-K] v^{m},
$$

where we have dropped the tildes. Therefore, throughout this paper, we always assume that $d_{1}=d, d_{2}=1, m>1, c>0$ and $u_{0} \in\left(0, u_{K}\right)$. Hence a traveling wave solution $(u, v)$ of (1.1) with wave speed $c$ satisfies the system of differential equations

$$
d u^{\prime \prime}+c u^{\prime}-f(u) v^{m}=0, \quad v^{\prime \prime}+c v^{\prime}+[f(u)-K] v^{m}=0,
$$

subject to the boundary conditions

$$
\begin{aligned}
\lim _{t \rightarrow-\infty}\left(u, u^{\prime}, v, v^{\prime}\right) & =\left(u_{0}, 0,0,0\right), \\
\lim _{t \rightarrow+\infty}\left(u, u^{\prime}, v, v^{\prime}\right) & =\left(u^{0}, 0,0,0\right)
\end{aligned}
$$

for some $u_{0} \in\left(0, u_{K}\right)$ and $u^{0}>u_{K}$. Here the prime denotes $d / d t$.

2.1. Reduction to the first-order system. By setting the variables

$$
u_{1}=u, \quad u_{2}=u^{\prime}, \quad v_{1}=v, \quad v_{2}=v^{\prime},
$$

then the problem (2.1)-(2.2a) is converted to the following (initial value) problem $\left(P_{u_{0}}\right)$ :

$$
\begin{aligned}
& \left(\begin{array}{c}
u_{1}^{\prime} \\
u_{2}^{\prime} \\
v_{1}^{\prime} \\
v_{2}^{\prime}
\end{array}\right)=\left(\begin{array}{c}
u_{2} \\
{\left[-c u_{2}+f\left(u_{1}\right) v_{1}^{m}\right] / d} \\
v_{2} \\
-c v_{2}+\left[K-f\left(u_{1}\right)\right] v_{1}^{m}
\end{array}\right), \quad v_{1}>0, \\
& \lim _{t \rightarrow-\infty}\left(u_{1}(t), u_{2}(t), v_{1}(t), v_{2}(t)\right)=\left(u_{0}, 0,0,0\right) .
\end{aligned}
$$

In the remainder of this section, we will establish the uniqueness and existence of solutions of the problem $\left(P_{u_{0}}\right)$ for each given $u_{0} \in\left(0, u_{K}\right)$ and $c>0$. More precisely, the uniqueness and local existence of solutions of $\left(P_{u_{0}}\right)$ are presented in subsection 2.2. Then in subsection 2.3, we will derive some useful integral identities and basic properties of solutions of $\left(P_{u_{0}}\right)$. Finally, subsection 2.4 is devoted to the global existence of solutions of $\left(P_{u_{0}}\right)$. 
2.2. Local existence and uniqueness of solutions of $\left(P_{u_{0}}\right)$. We begin with the investigation of the local dynamics of (2.3) near the equilibrium point $\left(u_{0}, 0,0,0\right)$ with $u_{0} \in\left(0, u_{K}\right)$. Indeed, a simple computation shows that the coefficient matrix for the linearized system of (2.3) at $\left(u_{0}, 0,0,0\right)$ with $u_{0}>0$ is given by

$$
A=\left(\begin{array}{cccc}
0 & 1 & 0 & 0 \\
0 & -c / d & 0 & 0 \\
0 & 0 & 0 & 1 \\
0 & 0 & 0 & -c
\end{array}\right)
$$

and the eigenvalues of $A$ are

$$
\lambda_{1}=\lambda_{2}=0, \lambda_{3}=-c / d, \text { and } \lambda_{4}=-c,
$$

whose corresponding eigenvectors are

$$
\mathbf{q}_{1}=\left(\begin{array}{l}
1 \\
0 \\
0 \\
0
\end{array}\right), \quad \mathbf{q}_{2}=\left(\begin{array}{l}
0 \\
0 \\
1 \\
0
\end{array}\right), \quad \mathbf{q}_{3}=\left(\begin{array}{c}
1 \\
-c / d \\
0 \\
0
\end{array}\right), \quad \text { and } \mathbf{q}_{4}=\left(\begin{array}{c}
0 \\
0 \\
1 \\
-c
\end{array}\right),
$$

respectively. Note that the resulting linearized system is independent of the order of the reaction $m$ (if $m>1$ ) and the choice of the equilibrium point $\left(u_{0}, 0,0,0\right)$. Hence it follows from the standard theory (see Carr [7]) that (2.3) has a two-dimensional local centre manifold $\mathcal{W}^{c}$ and a two-dimensional local stable manifold $\mathcal{W}^{u}$ near the equilibrium point $\left(u_{0}, 0,0,0\right)$ with $u_{0}>0$. This implies that any solution of the problem $\left(P_{u_{0}}\right)$ (if it exists) would lie on a two-dimensional local centre manifold $\mathcal{W}^{c}$.

Now we employ the change of independent variables

$$
\left(\begin{array}{c}
u_{1}-u_{0} \\
u_{2} \\
v_{1} \\
v_{2}
\end{array}\right):=\left[\mathbf{q}_{1}, \mathbf{q}_{2}, \mathbf{q}_{3}, \mathbf{q}_{4}\right]\left(\begin{array}{c}
x_{1} \\
x_{2} \\
x_{3} \\
x_{4}
\end{array}\right)
$$

This transforms the problem $\left(P_{u_{0}}\right)$ into the following (initial value) problem $\left(\hat{P}_{u_{0}}\right)$ :

$$
\begin{aligned}
\left(\begin{array}{l}
x_{1}^{\prime} \\
x_{2}^{\prime} \\
x_{3}^{\prime} \\
x_{4}^{\prime}
\end{array}\right)= & \left(\begin{array}{cccc}
0 & 0 & 0 & 0 \\
0 & 0 & 0 & 0 \\
0 & 0 & -c / d & 0 \\
0 & 0 & 0 & -c
\end{array}\right) \cdot\left(\begin{array}{l}
x_{1} \\
x_{2} \\
x_{3} \\
x_{4}
\end{array}\right) \\
& +\left(\begin{array}{c}
\frac{1}{c} f\left(x_{1}+x_{3}+u_{0}\right) \cdot\left(x_{2}+x_{4}\right)^{m} \\
\frac{1}{c}\left[K-f\left(x_{1}+x_{3}+u_{0}\right)\right]\left(x_{2}+x_{4}\right)^{m} \\
-\frac{1}{c} f\left(x_{1}+x_{3}+u_{0}\right) \cdot\left(x_{2}+x_{4}\right)^{m} \\
-\frac{1}{c}\left[K-f\left(x_{1}+x_{3}+u_{0}\right)\right]\left(x_{2}+x_{4}\right)^{m}
\end{array}\right), \quad x_{2}+x_{4}>0, \\
& \lim _{t \rightarrow-\infty}\left(x_{1}(t), x_{2}(t), x_{3}(t), x_{4}(t)\right)=(0,0,0,0),
\end{aligned}
$$

and correspondingly transforms any local centre manifold of (2.3) at $\left(u_{0}, 0,0,0\right)$ into a local centre manifold of (2.4) at the origin, which is tangent to the $\left(x_{1}, x_{2}\right)$-plane and 
can be represented by a surface

$$
\begin{array}{r}
\mathcal{W}_{\left(\psi_{1}, \psi_{2}\right)}^{c}(\mathbf{0}):=\left\{\left(x_{1}, x_{2}, x_{3}, x_{4}\right) \in \mathbb{R}^{4} \mid x_{3}=\psi_{1}\left(x_{1}, x_{2}\right), x_{4}=\psi_{2}\left(x_{1}, x_{2}\right), x_{2}+x_{4}>0\right. \\
\text { for } \left.|\mathbf{x}|<\delta_{0}\right\}
\end{array}
$$

for some smooth functions $\psi_{i}$ with $\psi_{i}(0,0)=\psi_{i, x_{1}}(0,0)=\psi_{i, x_{2}}(0,0)=0, i=1,2$, and sufficiently small $\delta_{0}$. Here we denote $\mathbf{x}=\left(x_{1}, x_{2}, x_{3}, x_{4}\right)^{t}$ and $|\mathbf{x}|=\sqrt{x_{1}^{2}+x_{2}^{2}+x_{3}^{2}+x_{4}^{2}}$.

Next we shall use the centre manifold theory (see Carr [7]) to derive the asymptotic expansion of any centre manifold $\mathcal{W}_{\left(\psi_{1}, \psi_{2}\right)}^{c}(\mathbf{0})$ of (2.4) near the origin which can be characterized by $\left(\psi_{1}, \psi_{2}\right)$. We first denote $\hat{\mathbf{x}}=\left(x_{1}, x_{2}\right)^{t}$ and $|\hat{\mathbf{x}}|=\sqrt{x_{1}^{2}+x_{2}^{2}}$. Then for all $\hat{\mathbf{x}}$ with $|\hat{\mathbf{x}}| \ll 1$, it follows from the centre manifold theory that

$$
\begin{aligned}
& \left(\begin{array}{cc}
\psi_{1, x_{1}} & \psi_{1, x_{2}} \\
\psi_{2, x_{1}} & \psi_{2, x_{2}}
\end{array}\right) \cdot\left(\begin{array}{c}
\frac{1}{c} f\left(x_{1}+\psi_{1}+u_{0}\right) \cdot\left(x_{2}+\psi_{2}\right)^{m} \\
\frac{1}{c}\left[K-f\left(x_{1}+\psi_{1}+u_{0}\right)\right]\left(x_{2}+\psi_{2}\right)^{m}
\end{array}\right) \\
= & \left(\begin{array}{c}
-\frac{c}{d} \psi_{1} \\
-c \psi_{2}
\end{array}\right)+\left(\begin{array}{c}
-\frac{1}{c} f\left(x_{1}+\psi_{1}+u_{0}\right) \cdot\left(x_{2}+\psi_{2}\right)^{m} \\
-\frac{1}{c}\left[K-f\left(x_{1}+\psi_{1}+u_{0}\right)\right]\left(x_{2}+\psi_{2}\right)^{m}
\end{array}\right),
\end{aligned}
$$

which is equivalent to

$$
\begin{aligned}
- & \frac{c}{d} \psi_{1}-f\left(x_{1}+\psi_{1}+u_{0}\right) \frac{\left(x_{2}+\psi_{2}\right)^{m}}{c} \\
& =\psi_{1, x_{1}} f\left(x_{1}+\psi_{1}+u_{0}\right) \frac{\left(x_{2}+\psi_{2}\right)^{m}}{c}+\psi_{1, x_{2}}\left[K-f\left(x_{1}+\psi_{1}+u_{0}\right)\right] \frac{\left(x_{2}+\psi_{2}\right)^{m}}{c}, \\
- & c \psi_{2}-\left[K-f\left(x_{1}+\psi_{1}+u_{0}\right)\right] \frac{\left(x_{2}+\psi_{2}\right)^{m}}{c} \\
& =\psi_{2, x_{1}} f\left(x_{1}+\psi_{1}+u_{0}\right) \frac{\left(x_{2}+\psi_{2}\right)^{m}}{c}+\psi_{2, x_{2}}\left[K-f\left(x_{1}+\psi_{1}+u_{0}\right)\right] \frac{\left(x_{2}+\psi_{2}\right)^{m}}{c} .
\end{aligned}
$$

Here for $i=1,2$, we write $\partial \psi_{i} / \partial x_{1}$ and $\partial \psi_{i} / \partial x_{2}$ as $\psi_{i, x_{1}}$ and $\psi_{i, x_{2}}$, respectively, and ignore the arguments of $\psi_{i}, \psi_{i, x_{1}}$, and $\psi_{i, x_{2}}$. Since (2.5)-(2.6) are identities for all $\hat{\mathbf{x}}$ with $|\hat{\mathbf{x}}| \ll 1$, it follows that

$$
\begin{aligned}
& \psi_{1}\left(x_{1}, x_{2}\right)=-d\left(f\left(u_{0}\right)+O(|\hat{\mathbf{x}}|)\right) \frac{\left(x_{2}+\psi_{2}\right)^{m}}{c^{2}}, \\
& \psi_{2}\left(x_{1}, x_{2}\right)=-\left(K-f\left(u_{0}\right)+O(|\hat{\mathbf{x}}|)\right) \frac{\left(x_{2}+\psi_{2}\right)^{m}}{c^{2}} .
\end{aligned}
$$

From (2.8) and $m>1$, we see that $\psi_{2}\left(x_{1}, 0\right)=0$ for all $x_{1}$ with $\left|x_{1}\right| \ll 1$. Hence by using the mean-value theorem, we have that

$$
\psi_{2}\left(x_{1}, x_{2}\right)=\psi_{2}\left(x_{1}, x_{2}\right)-\psi_{2}\left(x_{1}, 0\right)=x_{2} O(|\hat{\mathbf{x}}|)
$$

for all $\hat{\mathbf{x}}$ with $|\hat{\mathbf{x}}| \ll 1$, which, together with (2.7)-(2.8), gives

$$
\begin{aligned}
& \psi_{1}\left(x_{1}, x_{2}\right)=-\frac{d}{c^{2}} f\left(u_{0}\right) x_{2}{ }^{m} \cdot(1+O(|\hat{\mathbf{x}}|)), \\
& \psi_{2}\left(x_{1}, x_{2}\right)=-\frac{K-f\left(u_{0}\right)}{c^{2}} x_{2}{ }^{m} \cdot(1+O(|\hat{\mathbf{x}}|))
\end{aligned}
$$

for all $\hat{\mathbf{x}}$ with $|\hat{\mathbf{x}}| \ll 1$ and $x_{2}>0$. Hence $x_{2}+\psi_{2}\left(x_{1}, x_{2}\right)>0$ for all $\hat{\mathbf{x}}$ with $|\hat{\mathbf{x}}| \ll 1$ and $x_{2}>0$. Notice that the leading terms in (2.9) and (2.10) are independent of the choice $\left(\psi_{1}, \psi_{2}\right)$. 
Lemma 2.1. For each $c>0$ and $u_{0} \in\left(0, u_{K}\right)$, there exists a unique solution $(u, v)$ (up to a translation) of (2.1) which is defined on $(-\infty, S)$ for some $S \in \mathbb{R}$, such that the following hold:

(i) $u(t)>u_{0}, u^{\prime}(t)>0, v(t)>0$ and $v^{\prime}(t)>0$ for all $t \in(-\infty, S)$.

(ii) $\lim _{t \rightarrow-\infty}\left(u(t), u^{\prime}(t), v(t), v^{\prime}(t)\right)=\left(u_{0}, 0,0,0\right)$.

Proof. Step 1. We first prove the existence of $(u, v)$ with the desired properties. To this end we first consider the problem $\left(\hat{P}_{u_{0}}\right)$. Fix a pair of smooth functions $\left(\psi_{1}, \psi_{2}\right)$ defining a local centre manifold $\mathcal{W}_{\left(\psi_{1}, \psi_{2}\right)}^{c}(\mathbf{0})$ of (2.4) at the origin. In order to solve the problem $\left(\hat{P}_{u_{0}}\right)$, we claim that there is a solution $\left(x_{1}(t), x_{2}(t), x_{3}(t), x_{4}(t)\right)$ of (2.4) which lies on this local centre manifold $\mathcal{W}_{\left(\psi_{1}, \psi_{2}\right)}^{c}(\mathbf{0})$ and satisfies the boundary condition $\lim _{t \rightarrow-\infty}\left(x_{1}(t), x_{2}(t)\right)=(0,0)$. We first observe that by the centre manifold theory (see Carr [7), the first two components $\left(x_{1}(t), x_{2}(t)\right)$ of such a solution $\left(x_{1}(t), x_{2}(t), x_{3}(t), x_{4}(t)\right)$ give a solution of the following problem $\left(Q_{u_{0}}\right)$ :

$$
\begin{aligned}
& x_{1}^{\prime}=f\left(x_{1}+\psi_{1}\left(x_{1}, x_{2}\right)+u_{0}\right) \cdot\left(x_{2}+\psi_{2}\left(x_{1}, x_{2}\right)\right)^{m} / c, \\
& x_{2}^{\prime}=\left[K-f\left(x_{1}+\psi_{1}\left(x_{1}, x_{2}\right)+u_{0}\right)\right] \cdot\left(x_{2}+\psi_{2}\left(x_{1}, x_{2}\right)\right)^{m} / c
\end{aligned}
$$

subject to the conditions

$$
\lim _{t \rightarrow-\infty}\left(x_{1}(t), x_{2}(t)\right)=(0,0), \quad x_{2}(t)+\psi_{2}\left(x_{1}(t), x_{2}(t)\right)>0 .
$$

Note that the problem $\left(Q_{u_{0}}\right)$ can be reduced to the following initial value problem $\left(\widehat{Q}_{u_{0}}\right)$ :

$$
\begin{aligned}
\frac{d x_{2}}{d x_{1}} & =\frac{K-f\left(x_{1}+\psi_{1}\left(x_{1}, x_{2}\right)+u_{0}\right)}{f\left(x_{1}+\psi_{1}\left(x_{1}, x_{2}\right)+u_{0}\right)}, \quad x_{2}+\psi_{2}\left(x_{1}, x_{2}\right)>0, \\
x_{2}(0) & =0 .
\end{aligned}
$$

Furthermore, if $|\hat{\mathbf{x}}| \ll 1$, then $\left(\widehat{Q}_{u_{0}}\right)$ is equivalent to the problem

$$
\frac{d x_{2}}{d x_{1}}=\frac{K-f\left(u_{0}\right)}{f\left(u_{0}\right)}+R\left(x_{1}, x_{2}\right), \quad x_{2}+\psi_{2}\left(x_{1}, x_{2}\right)>0, x_{2}(0)=0,
$$

where $R\left(x_{1}, x_{2}\right)$ is smooth in $x_{1}$ and $x_{2}$ and $R\left(x_{1}, x_{2}\right)=O(|\hat{\mathbf{x}}|)$, and $\hat{\mathbf{x}}=\left(x_{1}, x_{2}\right)^{t}$. Using this reduced form and noting the expression (2.10) for $\psi_{2}$, it follows that there exists a unique solution $x_{2}=h\left(x_{1}\right)$ of $\left(\widehat{Q}_{u_{0}}\right)$ defined on $\left[0, x_{0}\right]$ for some sufficiently small $x_{0}>0$ such that $h(0)=0, h^{\prime}(0)=\left(K-f\left(u_{0}\right)\right) / f\left(u_{0}\right)$, and $h\left(x_{1}\right)>0$ and $h\left(x_{1}\right)+\psi_{2}\left(x_{1}, h\left(x_{1}\right)\right)>0$ for all $x \in\left(0, x_{0}\right]$.

From the above discussion, we can conclude that there exists a unique solution $\left(x_{1}(t), x_{2}(t)\right)$ (up to a translation) of (2.11) defined for all $t \in(-\infty, 0]$ (with a suitable shift of $t$ ) such that $x_{1}>0, x_{2}>0$, and $x_{2}(t)+\psi_{2}\left(x_{1}(t), x_{2}(t)\right)>0$ on $(-\infty, 0]$, and $\lim _{t \rightarrow-\infty}\left(x_{1}(t), x_{2}(t)\right)=(0,0)$. Now by setting $x_{3}(t)=\psi_{1}\left(x_{1}(t), x_{2}(t)\right)$ and $x_{4}(t)=$ $\psi_{2}\left(x_{1}(t), x_{2}(t)\right)$, it follows that there is a solution $\left(x_{1}(t), x_{2}(t), x_{3}(t), x_{4}(t)\right)$ of the problem $\left(\hat{P}_{u_{0}}\right)$ defined on $(-\infty, 0]$ (with a suitable shift of $t$ if necessary) such that $x_{1}>0, x_{2}>0$, $x_{3}<0, x_{4}<0$, and $x_{2}+x_{4}>0$ on $(-\infty, 0]$, and $\lim _{t \rightarrow-\infty}\left(x_{1}(t), x_{2}(t), x_{3}(t), x_{4}(t)\right)=$ $(0,0,0,0)$. Finally, by transferring back to the origin the variables $\left(u, u^{\prime}, v, v^{\prime}\right)$ and noting that $u^{\prime}=-c x_{3} / d, v=x_{2}+x_{4}$ and $v^{\prime}=-c x_{4}$, we then obtain that there exists a solution $\left(u(t), u^{\prime}(t), v(t), v^{\prime}(t)\right)$ of the problem $\left(P_{u_{0}}\right)$ defined on $(-\infty, T)$ for some $T \in \mathbb{R}$ such 
that $u>u_{0}, u^{\prime}>0, v>0$ and $v^{\prime}>0$ on $(-\infty, T)$, and $\lim _{t \rightarrow-\infty}\left(u(t), u^{\prime}(t), v(t), v^{\prime}(t)\right)=$ $\left(u_{0}, 0,0,0\right)$. Hence we establish the existence of $(u, v)$ with the desired properties.

Step 2. Next, we need to show the uniqueness of the solution to $\left(P_{u_{0}}\right)$. We shall use the method of Lemma 2.1 of Ai and Huang 2 to prove it. Recall that the problems $\left(P_{u_{0}}\right)$ and $\left(\hat{P}_{u_{0}}\right)$ are equivalent. Thus it suffices to show the uniqueness of solutions to $\left(\hat{P}_{u_{0}}\right)$. To this end we first observe that any solution $\left(x_{1}(t), x_{2}(t), x_{3}(t), x_{4}(t)\right)$ of $\left(\hat{P}_{u_{0}}\right)$ must lie on a local centre manifold $\mathcal{W}_{\left(\psi_{1}, \psi_{2}\right)}^{c}(\mathbf{0})$ of (2.4) at the origin for negative $t$ with, $|t| \gg 1$, and any solution $\left(x_{1}(t), x_{2}(t), x_{3}(t), x_{4}(t)\right)$ of $\left(\hat{P}_{u_{0}}\right)$ lying on $\mathcal{W}_{\left(\psi_{1}, \psi_{2}\right)}^{c}(\mathbf{0})$ for negative $t$ with $|t| \gg 1$ is unique up to a translation. With this observation, it suffices to show that $\left(x_{1}(t), x_{2}(t), x_{3}(t), x_{4}(t)\right)$ is independent of the choice of smooth functions $\left(\psi_{1}, \psi_{2}\right)$ characterizing the local centre manifold $\mathcal{W}_{\left(\psi_{1}, \psi_{2}\right)}^{c}(\mathbf{0})$ of (2.4) at the origin.

To begin with, we suppose that $\left(\psi_{1}, \psi_{2}\right)$ and $\left(\hat{\psi}_{1}, \hat{\psi}_{2}\right)$ are the representations of two local centre manifolds of (2.4) at $(0,0,0,0)$. Let $\left(x_{1}(t), x_{2}(t)\right)$ be the solution of (2.11) with $\left(\psi_{1}, \psi_{2}\right)$ constructed in the previous paragraph. Therefore, with the use of (2.9)(2.10) and a suitable shift of $t,\left(x_{1}(t), x_{2}(t), x_{3}(t), x_{4}(t)\right):=\left(x_{1}(t), x_{2}(t), \psi_{1}\left(x_{1}(t), x_{2}(t)\right)\right.$, $\left.\psi_{2}\left(x_{1}(t), x_{2}(t)\right)\right)$ is a solution of the problem $\left(\hat{P}_{u_{0}}\right)$ defined on $(-\infty, 0]$. Note that $\hat{\psi}_{1}\left(x_{1}(t), x_{2}(t)\right)$ and $\hat{\psi}_{2}\left(x_{1}(t), x_{2}(t)\right)$ are defined for negative $t$ with $|t| \gg 1$. We now claim that $x_{3}(t)=\hat{\psi}_{1}\left(x_{1}(t), x_{2}(t)\right)$ and $x_{4}(t)=\hat{\psi}_{2}\left(x_{1}(t), x_{2}(t)\right)$ for negative $t$ with $|t| \gg 1$. For simplicity we set $\eta_{1}(t):=x_{3}(t)-\hat{\psi}_{1}\left(x_{1}(t), x_{2}(t)\right)$ and $\eta_{2}(t):=x_{4}(t)-$ $\hat{\psi}_{2}\left(x_{1}(t), x_{2}(t)\right)$ for negative $t$ with $|t| \gg 1$, write $\partial \psi_{i} / \partial x_{1}$ and $\partial \psi_{i} / \partial x_{2}$ as $\psi_{i, x_{1}}$ and $\psi_{i, x_{2}}$, respectively, and ignore the arguments of $\psi_{i}, \psi_{i, x_{1}}, \psi_{i, x_{2}}$. Recall from (2.5)-(2.6) that $\left(\hat{\psi}_{1}\left(x_{1}, x_{2}\right), \hat{\psi}_{2}\left(x_{1}, x_{2}\right)\right)$ satisfies

$$
\begin{aligned}
& \hat{\psi}_{1, x_{1}} f\left(x_{1}+\hat{\psi}_{1}+u_{0}\right) \frac{\left(x_{2}+\hat{\psi}_{2}\right)^{m}}{c}+\hat{\psi}_{1, x_{2}}\left[K-f\left(x_{1}+\hat{\psi}_{1}+u_{0}\right)\right] \frac{\left(x_{2}+\hat{\psi}_{2}\right)^{m}}{c} \\
& =-\frac{c}{d} \hat{\psi}_{1}-f\left(x_{1}+\hat{\psi}_{1}\left(x_{1}, x_{2}\right)+u_{0}\right) \frac{\left(x_{2}+\hat{\psi}_{2}\right)^{m}}{c}, \\
& \hat{\psi}_{2, x_{1}} f\left(x_{1}+\hat{\psi}_{1}+u_{0}\right) \frac{\left(x_{2}+\hat{\psi}_{2}\right)^{m}}{c}+\hat{\psi}_{2, x_{2}}\left[K-f\left(x_{1}+\hat{\psi}_{1}+u_{0}\right)\right] \frac{\left(x_{2}+\hat{\psi}_{2}\right)^{m}}{c} \\
& =-c \hat{\psi}_{2}-\left[K-f\left(x_{1}+\hat{\psi}_{1}+u_{0}\right)\right] \frac{\left(x_{2}+\hat{\psi}_{2}\right)^{m}}{c}
\end{aligned}
$$

for all sufficiently small $x_{1}, x_{2}$ with $x_{2}+\hat{\psi}_{2}>0$. Also recall from (2.4) that $x_{3}(t)$ and $x_{4}(t)$ satisfy

$$
\begin{aligned}
& x_{3}^{\prime}(t)=-\frac{c}{d} x_{3}(t)-f\left(x_{1}(t)+x_{3}(t)+u_{0}\right) \cdot \frac{\left(x_{2}(t)+x_{4}(t)\right)^{m}}{c}, \\
& x_{4}^{\prime}(t)=-c x_{4}(t)-\left[K-f\left(x_{1}(t)+x_{3}(t)+u_{0}\right)\right] \cdot \frac{\left(x_{2}(t)+x_{4}(t)\right)^{m}}{c},
\end{aligned}
$$

for all $t \in(-\infty, 0]$. Subtracting (2.12) from (2.14), it yields

$$
\begin{aligned}
\eta_{1}^{\prime}= & -\frac{c}{d} \eta_{1}-f\left(x_{1}+x_{3}+u_{0}\right) \cdot \frac{\left(x_{2}+x_{4}\right)^{m}}{c}+f\left(x_{1}+\hat{\psi}_{1}+u_{0}\right) \cdot \frac{\left(x_{2}+\hat{\psi}_{2}\right)^{m}}{c} \\
& +\hat{\psi}_{1, x_{1}} f\left(x_{1}+\hat{\psi}_{1}+u_{0}\right) \cdot \frac{\left(x_{2}+\hat{\psi}_{2}\right)^{m}}{c}-\hat{\psi}_{1, x_{1}} f\left(x_{1}+x_{3}+u_{0}\right) \cdot \frac{\left(x_{2}+x_{4}\right)^{m}}{c}
\end{aligned}
$$




$$
\begin{aligned}
& +\hat{\psi}_{1, x_{2}}\left[K-f\left(x_{1}+\hat{\psi}_{1}+u_{0}\right)\right] \frac{\left(x_{2}+\hat{\psi}_{2}\right)^{m}}{c}-\hat{\psi}_{1, x_{2}}\left[K-f\left(x_{1}+x_{3}+u_{0}\right)\right] \frac{\left(x_{2}+x_{4}\right)^{m}}{c} \\
= & -\frac{c}{d} \eta_{1}-\left(f\left(x_{1}+x_{3}+u_{0}\right)-f\left(x_{1}+\hat{\psi}_{1}+u_{0}\right)\right) \frac{\left(x_{2}+x_{4}\right)^{m}}{c} \\
& -f\left(x_{1}+\hat{\psi}_{1}+u_{0}\right)\left(\frac{\left(x_{2}+x_{4}\right)^{m}}{c}-\frac{\left(x_{2}+\hat{\psi}_{2}\right)^{m}}{c}\right) \\
& -\hat{\psi}_{1, x_{1}} f\left(x_{1}+\hat{\psi}_{1}+u_{0}\right)\left(\frac{\left(x_{2}+x_{4}\right)^{m}}{c}-\frac{\left(x_{2}+\hat{\psi}_{2}\right)^{m}}{c}\right) \\
& -\hat{\psi}_{1, x_{1}}\left(f\left(x_{1}+x_{3}+u_{0}\right)-f\left(x_{1}+\hat{\psi}_{1}+u_{0}\right)\right) \cdot \frac{\left(x_{2}+x_{4}\right)^{m}}{c} \\
& -\hat{\psi}_{1, x_{2}}\left[K-f\left(x_{1}+\hat{\psi}_{1}+u_{0}\right)\right]\left(\frac{\left(x_{2}+x_{4}\right)^{m}}{c}-\frac{\left(x_{2}+\hat{\psi}_{2}\right)^{m}}{c}\right) \\
& +\hat{\psi}_{1, x_{2}}\left(f\left(x_{1}+x_{3}+u_{0}\right)-f\left(x_{1}+\hat{\psi}_{1}+u_{0}\right)\right) \cdot \frac{\left(x_{2}+x_{4}\right)^{m}}{c}
\end{aligned}
$$

for negative $t$ with $|t| \gg 1$, which, together with the use of the mean-value theorem, yields

$$
\eta_{1}^{\prime}(t)=-\frac{c}{d} \eta_{1}(t)+M_{1,1}(t) \eta_{1}(t)+M_{1,2}(t) \eta_{2}(t)
$$

for negative $t$ with $|t| \gg 1$. Here $M_{1, i}(t), i=1,2$, are bounded functions and $M_{1, i}(t) \rightarrow 0$ as $t \rightarrow-\infty$. Similarly, by subtracting (2.13) from (2.15), $\eta_{2}$ satisfies

$$
\eta_{2}^{\prime}(t)=-c \eta_{2}(t)+M_{2,1}(t) \eta_{1}(t)+M_{2,2}(t) \eta_{2}(t)
$$

for negative $t$ with $|t| \gg 1$, where $M_{2, i}(t), i=1,2$, are bounded functions and $M_{2, i}(t) \rightarrow 0$ as $t \rightarrow-\infty$. The remaining proof of this claim follows the step 2 of Lemma 2.1 in $\mathrm{Ai}$ and Huang [2]. It is easy to verify that $\eta_{1}$ and $\eta_{2}$ satisfy the following identities:

$$
\begin{aligned}
& \eta_{1}(t)=\int_{-\infty}^{t} e^{-c(t-s) / d}\left(M_{1,1}(s) \eta_{1}(s)+M_{1,2}(s) \eta_{2}(s)\right) d s, \\
& \eta_{2}(t)=\int_{-\infty}^{t} e^{-c(t-s)}\left(M_{2,1}(s) \eta_{1}(s)+M_{2,2}(s) \eta_{2}(s)\right) d s
\end{aligned}
$$

for negative $t$ with $|t| \gg 1$. Set $\sigma:=\min \{1,1 / d\}$. Since $M_{i, j}(t) \rightarrow 0$ as $t \rightarrow-\infty$ for $i, j \in\{1,2\}$, we can choose a negative $s_{0}$ with $\left|s_{0}\right| \gg 1$ such that $\left|M_{i, j}(s)\right| \leq \sigma c / 4$ for all $s \in\left(-\infty, s_{0}\right]$ and $i, j \in\{1,2\}$. Then in view of the above two equations and the boundedness of $\eta_{i}, i=1,2$, we have

$$
\begin{aligned}
\left|\eta_{1}(t)\right|+\left|\eta_{2}(t)\right| & \leq \frac{\sigma c}{2} \int_{-\infty}^{t} e^{-\sigma c(t-s)}\left(\left|\eta_{1}(s)\right|+\left|\eta_{2}(s)\right|\right) d s \\
& \leq \frac{1}{2} \sup _{s \in\left(-\infty, s_{0}\right]}\left(\left|\eta_{1}(s)\right|+\left|\eta_{2}(s)\right|\right)
\end{aligned}
$$

for all $t \in\left(-\infty, s_{0}\right]$, which leads to $\eta_{1}(t)=\eta_{2}(t)=0$ for all $t \in\left(-\infty, s_{0}\right]$. Together with the definition of $\eta_{i}(t), i=1,2$, we obtain that $x_{3}(t)=\hat{\psi}_{1}\left(x_{1}(t), x_{2}(t)\right)$ and $x_{4}(t)=$ $\hat{\psi}_{2}\left(x_{1}(t), x_{2}(t)\right)$ for negative $t$ with $|t| \gg 1$, thereby establishing the assertion of this claim. 
Hence it follows from the argument in the first paragraph of Step 1 that $\left(x_{1}(t), x_{2}(t)\right.$, $\left.\left.x_{3}(t), x_{2}(t)\right), x_{4}(t)\right)$ is the unique solution (up to a translation) of $\left(\hat{P}_{u_{0}}\right)$ lying on the local centre manifold $\mathcal{W}_{\left(\hat{\psi}_{,}, \hat{\psi}_{2}\right)}^{c}(\mathbf{0})$ for negative $t$ with $|t| \gg 1$. Therefore we can conclude that the construction of $\left(x_{1}(t), x_{2}(t), x_{3}(t), x_{4}(t)\right)$ is independent of the choice of smooth functions $\left(\psi_{1}, \psi_{2}\right)$ characterizing the local centre manifold $\mathcal{W}_{\left(\psi_{1}, \psi_{2}\right)}^{c}(\mathbf{0})$ of (2.4) at the origin. This proof of this lemma is thus completed.

2.3. Basic properties. For each given $u_{0} \in\left(0, u_{K}\right)$, recall from Lemma 2.1 that there is a unique solution $(u, v)$ (up to a translation) of (2.1) defined on $(-\infty, S)$ for some $S \in \mathbb{R}$ such that $u>0$ and $v>0$ on $(-\infty, S)$, and $\lim _{t \rightarrow-\infty}\left(u(t), u^{\prime}(t), v(t), v^{\prime}(t)\right)=\left(u_{0}, 0,0,0\right)$. Now for any $t_{0} \in(-\infty, S)$ and $t \in\left(t_{0}, S\right)$, by applying the variation of constant formula to system (2.1), we can derive the following useful integral identities (see Huang [17]):

$$
\begin{aligned}
u^{\prime}(t)= & u^{\prime}\left(t_{0}\right) e^{-c\left(t-t_{0}\right) / d}+\frac{1}{d} \int_{t_{0}}^{t} e^{-c(t-s) / d} f(u(s)) v^{m}(s) d s \\
u(t)= & u\left(t_{0}\right)+\frac{d}{c}\left[1-e^{-c\left(t-t_{0}\right) / d}\right] u^{\prime}\left(t_{0}\right) \\
& +\frac{1}{c} \int_{t_{0}}^{t}\left[1-e^{-c(t-s) / d}\right] f(u(s)) v^{m}(s) d s .
\end{aligned}
$$

Letting $t_{0} \rightarrow-\infty$ in (2.16a) - 2.17a $)$, we obtain that

$$
\begin{aligned}
u^{\prime}(t) & =\frac{1}{d} \int_{-\infty}^{t} e^{-c(t-s) / d} f(u(s)) v^{m}(s) d s \\
u(t) & =u_{0}+\frac{1}{c} \int_{-\infty}^{t}\left[1-e^{-c(t-s) / d}\right] f(u(s)) v^{m}(s) d s .
\end{aligned}
$$

Next, with the use of the above identities and a slight modification of the arguments in Lemma 2.2, Corollary 2.3, and Corollary 2.4 of Huang [17, we can state the basic properties of a solution of (2.1)-2.2a) as follows:

Lemma 2.2. Given $u_{0} \in\left(0, u_{K}\right)$, let $(u, v)$ be a solution of (2.1) and (2.2a), and $(-\infty, T)$ be the corresponding maximal interval on which $(u(t), v(t))$ exists and $v(t)$ is positive. Then we have the following:

(i) $\omega\left(u_{0}, c\right):=\lim _{t \rightarrow T^{-}} u(t)>u_{K}$ and $\lim _{t \rightarrow T^{-}} v(t)=0$;

(ii) $u, v, u^{\prime}$ and $v^{\prime}$ are bounded on $(-\infty, T)$;

(iii) there exists a positive number $C_{1}$ which depends only on $d$, such that

$$
0<v(t) \leq \frac{C_{1} K}{\inf _{u \in\left[u_{0}, \omega\left(u_{0}, c\right)\right]} f(u)} \cdot \omega\left(u_{0}, c\right) \text { for all } t \in(-\infty, T)
$$

(iv) there exist two numbers $t^{\prime}$ and $t_{1}$ with $t^{\prime}<t_{1}<T$ such that $u^{\prime}(t)>0$ for $t \in(-\infty, T), u\left(t^{\prime}\right)=u_{K}, v^{\prime}(t)>0$ for $t \in\left(-\infty, t_{1}\right)$, and $v^{\prime}(t)<0$ for $t \in\left(t_{1}, T\right)$.

2.4. Global existence of solutions of $\left(P_{u_{0}}\right)$. Now we come to the goal of this section. To begin with we define the function $l:\left(0, u_{K}\right) \times\left(u_{K},+\infty\right) \rightarrow \mathbb{R}^{+}$by

$$
l(a, b):=\left(\frac{C_{1} K b}{\inf _{u \in[a, b]} f(u)}\right)^{m-1},
$$


where $C_{1}$ is defined in part (iii) of Lemma 2.2. We note that $l(a, b)$ is a decreasing function of $a \in\left(0, u_{K}\right)$ and an increasing function of $b \in\left(u_{K},+\infty\right)$. We also need to define the function $\tilde{f}:\left[u_{K},+\infty\right) \rightarrow \mathbb{R}^{+}$by

$$
\tilde{f}(b):=\max _{u \in\left[u_{K}, b\right]} f(u) .
$$

Lemma 2.3. Given $u_{0} \in\left(0, u_{K}\right)$ and $u^{0}>u_{K}$, let $(u, v)$ be a solution of (2.1) and (2.2a), and let $(-\infty, T)$ be the corresponding maximal interval on which $(u(t), v(t))$ exists and $v(t)$ is positive. Suppose that $u^{0}=\lim _{t \rightarrow T^{-}} u(t)$ and the positive number $c$ satisfies

$$
c^{2}>4 l\left(u_{0}, u^{0}\right)\left[\widetilde{f}\left(u^{0}\right)-K\right] .
$$

Then $(u, v)$ is a traveling wave solution of (1.1) with wave speed $c$ connecting $\left(u_{0}, 0\right)$ and $\left(u^{0}, 0\right)$ such that $u^{\prime}>0$ and $v>0$ on $\mathbb{R}$.

Proof. The proof is similar to that in Lemma 2.5 of Huang [17] except the modification to allow $m>1$. By part (iv) of Lemma 2.2 there exist $t^{\prime}, t_{1} \in(-\infty, T)$ such that $t^{\prime}<t_{1}$, $f(u(t))>K$ for all $t \in\left(t^{\prime}, T\right), v^{\prime}>0$ on $\left(-\infty, t_{1}\right)$ and $v^{\prime}<0$ on $\left(t_{1}, T\right)$. Let $v_{1}(t)=v(t)$ and $v_{2}(t)=v^{\prime}(t)$. Therefore, $\left(v_{1}(t), v_{2}(t)\right)$ stays at the region $\left\{v_{1}>0, v_{2}<0\right\}$ for all $t \in\left(t_{1}, T\right)$. By part (iii) of Lemma 2.2, we have

$$
l\left(u_{0}, u^{0}\right) v_{1}(t) \geq v_{1}^{m}(t)
$$

for each $t \in(-\infty, T)$. Fix a sufficiently positive small $\epsilon$ such that

$$
c^{2}>4 l\left(u_{0}, u^{0}\right)\left[\widetilde{f}\left(u^{0}\right)-K\right]+4 \epsilon .
$$

Therefore, we can choose a positive $\beta$ with the property that

$$
\beta^{2}-c \beta+l\left(u_{0}, u^{0}\right)\left[\widetilde{f}\left(u^{0}\right)-K\right]+\epsilon=0 .
$$

Now consider the region

$$
\Omega:=\left\{\left(v_{1}, v_{2}\right): v_{1}>0,-\beta v_{1}<v_{2}<0\right\} .
$$

We claim that $\left(v_{1}(t), v_{2}(t)\right)$ stays at the region $\Omega$ for all $t \in\left(t_{1}, T\right)$. First, for $t>t_{1}$ and $t$ is close to $t_{1}$, it is clear that $\left(v_{1}(t), v_{2}(t)\right)$ lies in the region $\Omega$. Next, since $f(u(t))>K$ for all $t \in\left[t_{1}, T\right)$, from the fourth equation of (2.3) we have that $v_{2}^{\prime}(t)=[K-f(u(t))] v_{1}^{m}(t)<0$ if $t \in\left[t_{1}, T\right)$ and $v_{2}(t)=0$. This implies that $\left(v_{1}(t), v_{2}(t)\right)$ cannot leave $\Omega$ through the positive $v_{1}$-axis. Now it remains to show that $\left(v_{1}(t), v_{2}(t)\right)$ cannot leave $\Omega$ through the ray $L:=\left\{\left(v_{1}, v_{2}\right): v_{1}>0, v_{2}=-\beta v_{1}\right\}$. For a contradiction, we assume that $\left(v_{1}(t), v_{2}(t)\right)$ leaves $\Omega$ at the first time $t_{2}>t_{1}$ and $\left(v_{1}\left(t_{2}\right), v_{2}\left(t_{2}\right)\right)$ lies on the ray $L$. Note that

$$
\mathbf{n}=(\beta, 1)
$$


is the inward normal to the line $L$. Then with a straightforward computation, it follows that when $t=t_{2}$, we have

$$
\begin{aligned}
\left(v_{1}^{\prime}\left(t_{2}\right), v_{2}^{\prime}\left(t_{2}\right)\right) \cdot \mathbf{n} & =\beta v_{2}\left(t_{2}\right)-\left[f\left(u\left(t_{2}\right)\right)-K\right] v_{1}^{m}\left(t_{2}\right)-c v_{2}\left(t_{2}\right) \quad(\text { by (2.3) }) \\
& =-\beta(\beta-c) v_{1}\left(t_{2}\right)-\left[f\left(u\left(t_{2}\right)\right)-K\right] v_{1}^{m}\left(t_{2}\right) \quad\left(\text { since } v_{2}\left(t_{2}\right)=-\beta v_{1}\left(t_{2}\right)\right) \\
& \geq-\beta(\beta-c) v_{1}\left(t_{2}\right)-l\left(u_{0}, u^{0}\right)\left[f\left(u\left(t_{2}\right)\right)-K\right] v_{1}\left(t_{2}\right) \quad(\text { by (2.20) }) \\
& =v_{1}\left(t_{2}\right)\left(l\left(u_{0}, u^{0}\right)\left[\widetilde{f}\left(u^{0}\right)-f\left(u\left(t_{2}\right)\right)\right]+\epsilon\right)>0 \quad(\text { by (2.21) }),
\end{aligned}
$$

which is a contradiction, thereby establishing that $\left(v_{1}(t), v_{2}(t)\right)$ cannot leave $\Omega$ through the ray $L$. Hence $\left(v_{1}(t), v_{2}(t)\right)$ must stay at the region $\Omega$ for all $t \in\left(t_{1}, T\right)$.

Using this claim and noting that $v_{1}(t)>0$ and $v_{2}(t)=v^{\prime}(t)<0$ for $t \in\left(t_{1}, T\right)$, we can conclude that $T=+\infty$, and $v(t) \rightarrow 0$ as $t \rightarrow+\infty$ by part (i) of Lemma 2.2. Moreover, $u(t) \rightarrow u^{0}$ as $t \rightarrow+\infty$. This completes the proof.

3. Proof of main result. In the remainder of this paper, we will let $\left(u\left(t ; u_{0}, c\right)\right.$, $\left.v\left(t ; u_{0}, c\right)\right)$ be a unique solution of (2.1) subject to the boundary condition $\lim _{t \rightarrow-\infty}\left(u\left(t ; u_{0}, c\right), u^{\prime}\left(t ; u_{0}, c\right), v\left(t ; u_{0}, c\right), v^{\prime}\left(t ; u_{0}, c\right)\right)=\left(u_{0}, 0,0,0\right)$ with $u_{0} \in\left(0, u_{K}\right)$ and $u\left(0 ; u_{0}, c\right)=u_{K}$, and let $\left(-\infty, T_{u_{0}, c}\right)$ be the corresponding maximal interval on which $\left(u\left(t ; u_{0}, c\right), v\left(t ; u_{0}, c\right)\right)$ exists and $v(t)$ is positive. Note that such a solution was shown to exist in Section 2. We will also let

$$
\omega\left(u_{0}, c\right):=\lim _{t \rightarrow T_{u_{0}, c}^{-}} u\left(t ; u_{0}, c\right) .
$$

If there is no ambiguity, for the sake of simplicity, we will write $\left(u\left(t ; u_{0}, c\right), v\left(t ; u_{0}, c\right)\right)$ and $T_{u_{0}, c}$ as $(u(t), v(t))$ and $T$, respectively.

In subsection 3.1, we will establish several auxiliary lemmas for the proof of Theorem 1.1 in subsection 3.2.

3.1. Several auxiliary lemmas. Let $\xi^{*}$ be defined as in the assumption (A3).

Lemma 3.1. Fix $u^{0}>u_{K}$ and let $u_{0}^{*} \in\left(0, \xi^{*}\right)$ be such that for each $u_{0} \in\left(0, u_{0}^{*}\right]$, we have

$$
\int_{u_{0}}^{\xi^{*}} \frac{1}{f(u)} d u>\frac{u^{0}-u_{0}}{K}
$$

Then for each $u_{0} \in\left(0, u_{0}^{*}\right]$ with $c^{2}>4 l\left(u_{0}, u^{0}\right)\left[\widetilde{f}\left(u^{0}\right)-K\right]$, we have $\omega\left(u_{0}, c\right)>u^{0}$.

Proof. The proof is based on the fact that $l\left(u_{0}, u^{0}\right)\left[\widetilde{f}\left(u^{0}\right)-K\right]$ is an increasing function of $u^{0} \in\left(u_{K},+\infty\right)$, and a slight modification of Lemma 3.1 of Huang [17. Hence we omit it.

Lemma 3.2. Fix $u^{0}>u_{K}$. Then there is a $\delta_{0} \in\left(0, \min \left\{1 / 2,\left(u^{0}-u_{K}\right) / 4\right\}\right)$ such that for each $\delta \in\left(0, \delta_{0}\right)$, there exists a $\sigma \in(0, \delta / 2)$ with the property that if $(u(t), v(t))=$ $\left(u\left(t ; u_{0}, c\right), v\left(t ; u_{0}, c\right)\right)$ with $u_{0} \in\left(0, u_{K}\right)$ satisfies that

$$
\left|u\left(t_{0}\right)-u^{0}\right|<\sigma, \quad\left|u^{\prime}\left(t_{0}\right)\right|<\sigma, \quad v^{\prime}\left(t_{0}\right) \leq 0, \quad \sqrt{v^{2}\left(t_{0}\right)+\left[v^{\prime}\left(t_{0}\right)\right]^{2}}<\sigma,
$$

for some $t_{0} \in \mathbb{R}$ and

$$
v(t)>0
$$


as long as $(u(t), v(t))$ exists, then $(u(t), v(t))$ exists on $\left[t_{0},+\infty\right)$, and

$$
\left|u\left(t_{0}\right)-u^{0}\right|<\delta \text { for all } t \geq t_{0} \text { and } \lim _{t \rightarrow+\infty} v(t)=0 .
$$

Proof. In view of the continuity of $f$, we can find a small positive $\delta_{0} \in(0, \min \{1 / 2$, $\left.\left.\left(u^{0}-u_{K}\right) / 4\right\}\right)$ such that for each $\delta \in\left(0, \delta_{0}\right)$, we have

$$
\left|f(u)-f\left(u^{0}\right)\right|<\frac{c^{2}}{32}
$$

for all $u \in\left[u^{0}-\delta, u^{0}+\delta\right]$. Set

$$
M:=\max _{u \in\left[u^{0}-\delta, u^{0}+\delta\right]} f(u)
$$

and consider the following positive-definite quadratic form

$$
\mathbb{V}\left(v_{1}, v_{2}\right)=\left(\frac{c^{2}}{2}+\left[f\left(u^{0}\right)-K\right]\right) v_{1}^{2}+c v_{1} v_{2}+v_{2}^{2},
$$

which satisfies the following inequality:

$$
\mu_{1}\left(v_{1}^{2}+v_{2}^{2}\right) \leq \mathbb{V}\left(v_{1}, v_{2}\right) \leq \mu_{2}\left(v_{1}^{2}+v_{2}^{2}\right)
$$

for all $\left(v_{1}, v_{2}\right) \in \mathbb{R}^{2}$ and for some positive numbers $\mu_{1}$ and $\mu_{2}$. Now we give the definition of $\sigma$ as follows:

$$
\sigma:=\min \left\{\sqrt{\frac{\mu_{1}}{5 \mu_{2}}}, \frac{\delta}{2}, \frac{c \delta}{4 d}, \frac{c \sqrt{\mu_{1}^{m}} \mu_{3}}{8 M \sqrt{\mu_{2}}} \delta\right\}
$$

where

$$
\mu_{3}:=c 2^{-\frac{m+3}{2}} \mu_{2}^{-\frac{m+1}{2}} \cdot \min \left\{\min _{u \in\left[u^{0}-\delta, u^{0}+\delta\right]} f(u)-K, 1\right\} .
$$

Let $(u, v)$ be a solution of (2.1) and (2.2a) satisfying the assumptions of this lemma. Observe that $\left|u\left(t_{0}\right)-u^{0}\right|<\sigma \leq \delta / 2$ and $\sqrt{v^{2}\left(t_{0}\right)+\left[v^{\prime}\left(t_{0}\right)\right]^{2}}<\sigma \leq 1 / 4$. Hence by the continuity, we have $\left|u(t)-u^{0}\right|<\delta$ and $\sqrt{v^{2}(t)+\left[v^{\prime}(t)\right]^{2}}<1 / 2$ for $t>t_{0}$ with $t-t_{0}$ sufficiently small. To proceed with the proof, we claim that if $(u, v)$ exists on $\left[t_{0}, s_{1}\right)$ and satisfies that $\left|u(t)-u^{0}\right|<\delta$ and $\sqrt{v^{2}(t)+\left[v^{\prime}(t)\right]^{2}}<1$ for all $t \in\left[t_{0}, s_{1}\right)$, then $\left|u^{\prime}(t)\right|$ is bounded and $\sqrt{v^{2}(t)+\left[v^{\prime}(t)\right]^{2}}<1 / 2$ on $\left[t_{0}, s_{1}\right)$. To do this we first note that $u(t)>u_{K}$ and $v^{\prime}(t)<0$ for $t \in\left(t_{0}, s_{1}\right)$ by part (iv) of Lemma 2.2. Hence $f(u(t))-K>0$ for $t \in\left(t_{0}, s_{1}\right)$. Consider the function

$$
\hat{\mathbb{V}}(t):=\mathbb{V}\left(v_{1}(t), v_{2}(t)\right),
$$

where $v_{1}(t)=v(t)$ and $v_{2}(t)=v^{\prime}(t)$, and $t \in\left(t_{0}, s_{1}\right)$. With a simple computation and the use of (2.3), we have

$$
\begin{aligned}
\hat{\mathbb{V}}^{\prime}(t)= & -c[f(u(t))-K] v_{1}^{m+1}(t)+2[K-f(u(t))] v_{1}^{m}(t) v_{2}(t) \\
& +2\left[f\left(u^{0}\right)-K\right] v_{1}(t) v_{2}(t)-c v_{2}^{2}(t) .
\end{aligned}
$$

Now we focus on the interval $t \in\left(t_{0}, s_{1}\right)$ and distinguish the following two cases:

(i) $v_{1}(t) \geq 4\left|v_{2}(t)\right| / c$;

(ii) $v_{1}(t)<4\left|v_{2}(t)\right| / c$. 
For case (i), we have $c v_{1}^{m+1}(t) / 2 \geq 2 v_{1}^{m}(t)\left|v_{2}(t)\right|$, which yields

$$
\begin{aligned}
\hat{\mathbb{V}}^{\prime}(t) & \leq-\frac{c}{2}[f(u(t))-K] v_{1}^{m+1}(t)+2\left[f\left(u^{0}\right)-K\right] v_{1}(t) v_{2}(t)-c v_{2}^{2}(t) \\
& \leq-\frac{c}{2}[f(u(t))-K] v_{1}^{m+1}(t)-\frac{c}{2} v_{2}^{2}(t) \quad\left(\text { since } v_{2}(t) \leq 0\right) .
\end{aligned}
$$

For case (ii), from (3.2) we have $c v_{2}^{2}(t) / 2>2\left|f(u(t))-f\left(u^{0}\right)\right| \cdot v_{1}(t)\left|v_{2}(t)\right|$. Hence we have that

$$
\begin{aligned}
\hat{\mathbb{V}}^{\prime}(t) \leq & -c[f(u(t))-K] v_{1}^{m+1}(t)+2[K-f(u(t))] v_{1}(t) v_{2}(t) \\
& +2\left[f\left(u^{0}\right)-K\right] v_{1}(t) v_{2}(t)-c v_{2}^{2}(t) \quad\left(\text { since } v_{1}(t) \in(0,1) \text { and } v_{2}(t)<0\right) \\
\leq & -c[f(u(t))-K] v_{1}^{m+1}(t)+2 v_{1}(t)\left|v_{2}(t)\right| \cdot\left|f(u(t))-f\left(u^{0}\right)\right|-c v_{2}^{2}(t) \\
\leq & -\frac{c}{2}[f(u(t))-K] v_{1}^{m+1}(t)-\frac{c}{2} v_{2}^{2}(t) .
\end{aligned}
$$

Hence both cases lead to

$$
\hat{\mathbb{V}}^{\prime}(t) \leq-\frac{c}{2}[f(u(t))-K] v_{1}^{m+1}(t)-\frac{c}{2} v_{2}^{2}(t)
$$

for all $t \in\left[t_{0}, s_{1}\right)$. For convenience we set

$$
\mu:=\frac{c}{2} \min \left\{\min _{u \in\left[u^{0}-\delta, u^{0}+\delta\right]} f(u)-K, 1\right\} .
$$

Then we have

$$
\begin{aligned}
\hat{\mathbb{V}}^{\prime}(t) \leq & -\frac{c}{2}[f(u(t))-K] v_{1}^{m+1}(t)-\frac{c}{2} v_{2}^{2}(t) \\
\leq & -\mu\left(v_{1}^{m+1}(t)+v_{2}^{2}(t)\right) \\
\leq & -\mu\left(v_{1}^{m+1}(t)+\left|v_{2}(t)\right|^{m+1}\right) \quad\left(\text { since }\left|v_{2}(t)\right| \in[0,1] \text { and } m>1\right) \\
\leq & -\frac{\mu}{2^{\frac{m+1}{2}}}\left(v_{1}^{2}(t)+v_{2}^{2}(t)\right)^{\frac{m+1}{2}} \\
& \left(\text { since }(a+b)^{p} \leq 2^{p}\left(a^{p}+b^{p}\right) \text { for all } a, b \geq 0 \text { and } p \geq 1\right) \\
\leq & -\mu_{3} \hat{\mathbb{V}}^{\frac{m+1}{2}}(t) \quad\left(\text { by }(\underline{3.3}) \text { and the definition of } \mu_{3}\right)
\end{aligned}
$$

for all $t \in\left[t_{0}, s_{1}\right)$, from which it follows that $\hat{\mathbb{V}}^{\prime}(t) \leq 0$ and

$$
\hat{\mathbb{V}}(t) \leq\left(\hat{\mathbb{V}} \frac{1-m}{2}\left(t_{0}\right)+\frac{\mu_{3}(m-1)}{2}\left(t-t_{0}\right)\right)^{\frac{2}{1-m}}
$$

for all $t \in\left[t_{0}, s_{1}\right)$. Together with (3.3) and the fact that $\sqrt{v^{2}\left(t_{0}\right)+\left[v^{\prime}\left(t_{0}\right)\right]^{2}}<\sigma \leq \sqrt{\frac{\mu_{1}}{5 \mu_{2}}}$, this yields

$$
\sqrt{v^{2}(t)+\left[v^{\prime}(t)\right]^{2}} \leq \sqrt{\frac{\hat{\mathbb{V}}\left(t_{0}\right)}{\mu_{1}}} \leq \sqrt{\frac{\mu_{2}}{\mu_{1}}} \cdot \sqrt{v^{2}\left(t_{0}\right)+\left[v^{\prime}\left(t_{0}\right)\right]^{2}}<\frac{1}{2}
$$


for all $t \in\left[t_{0}, s_{1}\right)$. This establishes the assertion for $\sqrt{v^{2}(t)+\left[v^{\prime}(t)\right]^{2}}$. Now we use the identity (2.16a) and the inequalities (3.3)-(3.4) to estimate $\left|u^{\prime}(t)\right|$ on $\left[t_{0}, s_{1}\right)$ as follows:

$$
\begin{aligned}
\left|u^{\prime}(t)\right| & \leq\left|u^{\prime}\left(t_{0}\right)\right|+\frac{1}{d} \int_{t_{0}}^{t} e^{-c(t-s) / d} f(u(s)) v^{m}(s) d s \\
& \leq \sigma+\frac{M}{d \sqrt{\mu_{1}^{m}}} \int_{t_{0}}^{t}\left(\hat{\mathbb{V}}^{\frac{1-m}{2}}\left(t_{0}\right)+\frac{\mu_{3}(m-1)}{2}\left(s-t_{0}\right)\right)^{\frac{m}{1-m}} d s \\
& \leq \sigma+\frac{2 M}{d \sqrt{\mu_{1}^{m}} \mu_{3}} \sqrt{\hat{\mathbb{V}}\left(t_{0}\right)},
\end{aligned}
$$

which shows the assertion for $\left|u^{\prime}(t)\right|$ on $\left[t_{0}, s_{1}\right)$. This completes the proof of the claim.

Hence if $(u(t), v(t))$ does not exist on $\left[t_{0},+\infty\right)$, then we can find an $s_{0}>t_{0}$ such that

$$
\left|u\left(s_{0}\right)-u^{0}\right|=\delta \text { and }\left|u(t)-u^{0}\right|<\delta \text { for all } t \in\left[t_{0}, s_{0}\right) .
$$

In what follows we will show that this leads to a contradiction. We first note that the inequality (3.4) still holds on $\left[t_{0}, s_{0}\right]$. Then we use the identity (2.17a) and the definition of $\sigma$ to obtain

$$
\begin{aligned}
\left|u\left(s_{0}\right)-u\left(t_{0}\right)\right| & \leq \frac{d}{c}\left|u^{\prime}\left(t_{0}\right)\right|+\frac{1}{c} \int_{t_{0}}^{s_{0}} f(u(s)) v^{m}(s) d s \\
& \leq \frac{d}{c} \sigma+\frac{M}{c \sqrt{\mu_{1}^{m}}} \int_{t_{0}}^{s_{0}}\left(\hat{\mathbb{V}}^{\frac{1-m}{2}}\left(t_{0}\right)+\frac{\mu_{3}(m-1)}{2}\left(s-t_{0}\right)\right)^{\frac{m}{1-m}} d s
\end{aligned}
$$

(by (3.3) and (3.4) )

$$
\begin{aligned}
& \leq \frac{d}{c} \sigma+\frac{2 M}{c \sqrt{\mu_{1}^{m}} \mu_{3}} \sqrt{\hat{\mathbb{V}}\left(t_{0}\right)} \\
& \leq \frac{d}{c} \sigma+\frac{2 M \sqrt{\mu_{2}}}{c \sqrt{\mu_{1}^{m}} \mu_{3}} \sqrt{v_{1}^{2}\left(t_{0}\right)+v_{2}^{2}\left(t_{0}\right)} \quad(\text { by }(\underline{3.3})) \\
& \leq \frac{d}{c} \sigma+\frac{2 M \sqrt{\mu_{2}}}{c \sqrt{\mu_{1}^{m}} \mu_{3}} \sigma<\frac{1}{2} \delta,
\end{aligned}
$$

which, together with the inequality $\left|u\left(t_{0}\right)-u^{0}\right|<\delta / 2$, yields

$$
\left|u\left(s_{0}\right)-u^{0}\right|<\delta \text {. }
$$

This is a contradiction. As a consequence $(u(t), v(t))$ exists on $\left[t_{0},+\infty\right)$ and $\left|u(t)-u^{0}\right|<\delta$

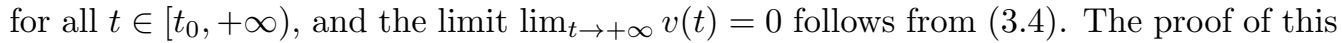
lemma is thus completed.

With a slight modification of the argument in Lemma 3.3 of Huang [17, we have the following lemma.

LEMma 3.3. There exists a $\bar{u}_{0} \in\left(0, u_{K}\right)$ such that for each $u_{0} \in\left[\bar{u}_{0}, u_{K}\right)$, we have

$$
0<v\left(t ; u_{0}, c\right) \leq u_{K}-u_{0}, \quad 0<v^{\prime}\left(t ; u_{0}, c\right) \leq \frac{K\left(u_{K}-u_{0}\right)^{m}}{c}
$$

for all $t \leq 0$.

In the remainder of this paper, we will retain the notation $\bar{u}_{0}$.

Recall the definition of $\omega\left(u_{0}, c\right)$ given in the beginning of this section. 
Lemma 3.4. For each fixed $u^{0}>u_{K}$ and $c^{2}>4 l\left(\bar{u}_{0}, u^{0}\right)\left[\widetilde{f}\left(u^{0}\right)-K\right]$, there exists a $\delta_{1}>0$ such that for each $u_{0} \in\left[\max \left\{\bar{u}_{0}, u_{K}-\delta_{1}\right\}, u_{K}\right)$, we have $\omega\left(u_{0}, c\right)<u^{0}$.

Proof. Consider the positive-definite quadratic form

$$
\mathbb{W}\left(v_{1}, v_{2}\right):=c^{2} v_{1}^{2}+2 c v_{1} v_{2}+2 v_{2}^{2}, \quad\left(v_{1}, v_{2}\right) \in \mathbb{R}^{2},
$$

which satisfies the following inequality:

$$
\rho_{1}\left(v_{1}^{2}+v_{2}^{2}\right) \leq \mathbb{W}\left(v_{1}, v_{2}\right) \leq \rho_{2}\left(v_{1}^{2}+v_{2}^{2}\right)
$$

for all $\left(v_{1}, v_{2}\right) \in \mathbb{R}^{2}$ and for some positive numbers $\rho_{1}$ and $\rho_{2}$. From the $\operatorname{limit}_{\lim _{u \rightarrow u_{K}}} f(u)$ $=K$, we can choose a $\bar{u} \in\left(u_{K}, u^{0}\right)$ with the property that

$$
\frac{c^{2}}{f(u)-K} \geq 5 \text { for all } u \in\left(u_{K}, \bar{u}\right] .
$$

Fix a positive $\delta<\min \left\{1, \delta_{0}, \bar{u}-u_{K}, u^{0}-\bar{u}\right\} / 2$, where $\delta_{0}$ is defined in Lemma 3.2 Let $\sigma$ be the number corresponding to $\delta_{0}$ in Lemma 3.2 and let $\bar{u}_{0}$ have the same definition as in Lemma 3.3. Recall that $\sigma \in(0, \delta / 2)$. Set

$$
M:=\max _{u \in\left[u_{K}, u^{0}\right]} f(u), \quad M_{1}:=\min \left\{\min _{u \in\left[\bar{u}, u^{0}\right]}(f(u)-K), 1\right\}, \quad \mu=2^{\frac{1-m}{2}} \rho_{2}^{-\frac{m+1}{2}} c M_{1}
$$

and

$$
\delta_{1}:=\min \left\{\frac{1}{2}, \frac{\sqrt{\rho_{1}} \cdot c \sigma}{\sqrt{\rho_{2}\left(K^{2}+c^{2}\right)}}, \frac{\sqrt{\rho_{1}} \cdot c^{2} \sigma}{\sqrt{\rho_{2}\left(K^{2}+c^{2}\right) \widetilde{f}\left(u^{0}\right)}}, \frac{1}{\left(\frac{d \widetilde{f}\left(u^{0}\right)}{c^{2}}+\frac{2 M \sqrt{\rho_{2}}}{c \sqrt{\rho_{1}^{m}} \mu}\right)} \sqrt{\frac{\rho_{1} c^{2}}{\rho_{2}\left(c^{2}+K^{2}\right)}} \cdot \frac{\sigma}{2}\right\} .
$$

We claim that for all $u_{0} \in\left[\max \left\{\bar{u}_{0}, u_{K}-\delta_{1}\right\}, u_{K}\right)$ with $c^{2}>4 l\left(\bar{u}_{0}, u^{0}\right)\left[\widetilde{f}\left(u^{0}\right)-u_{K}\right]$, we have $\omega\left(u_{0}, c\right)<u^{0}$. First since $\bar{u}<u^{0}$, we may assume that $\omega\left(u_{0}, c\right)>\bar{u}$. Recall the definition of $\left(u\left(t ; u_{0}, c\right), v\left(t ; u_{0}, c\right)\right)$. Also note that $u\left(0 ; u_{0}, c\right)=u_{K}$. For simplicity, we let $(u(t), v(t))=\left(u\left(t ; u_{0}, c\right), v\left(t ; u_{0}, c\right)\right)$. Then since $\omega\left(u_{0}, c\right)>\bar{u}$, we can choose a $t_{0}>0$ such that

$$
u(t) \in\left(u_{K}, \bar{u}\right) \text { for all } t \in\left(0, t_{0}\right) \text { and } u\left(t_{0}\right)=\bar{u} .
$$

From this it follows that $f(u(t))-K>0$ for all $t \in\left(0, t_{0}\right]$. Set $v_{1}(t)=v(t), v_{2}(t)=v^{\prime}(t)$, and $\hat{\mathbb{W}}(t)=\mathbb{W}\left(v_{1}(t), v_{2}(t)\right)$ for $t \in\left(0, t_{0}\right]$.

A straightforward calculation shows that

$$
\begin{aligned}
\hat{\mathbb{W}}^{\prime}(t) & =-\frac{2}{c}[f(u(t))-K]\left(c^{2} v_{1}^{m+1}(t)+2 c v_{1}^{m}(t) v_{2}(t)+\frac{c^{2}}{f(u(t))-K} v_{2}^{2}(t)\right) \\
& \left.\leq-\frac{2}{c}[f(u(t))-K]\left(c^{2} v_{1}^{m+1}(t)+2 c v_{1}^{m}(t) v_{2}(t)+5 v_{2}^{2}(t)\right) \text { (by (3.6) }\right)
\end{aligned}
$$

for all $t \in\left(0, t_{0}\right]$. We will show that $\hat{\mathbb{W}}^{\prime}(t)<0$ for all $t \in\left(0, t_{0}\right]$. To this end we recall from part (iv) of Lemma 2.2 that there is a $t_{1}>0$ such that $v_{2}(t)=v^{\prime}(t)>0$ for all $t \in\left(-\infty, t_{1}\right)$ and $v_{2}(t)=v^{\prime}(t)<0$ for all $t \in\left(t_{1}, T\right)$. Thus we need to consider the following two cases:

(A) $t_{1}<t_{0}$

(B) $t_{1} \geq t_{0}$. 
For case (A), we first claim that $\hat{\mathbb{W}}^{\prime}(t) \leq 0$ for all $t \in\left[0, t_{0}\right]$. Indeed, we have $v_{1}>0$ and $v_{2}>0$ on $\left[0, t_{1}\right)$. Hence from (3.7) it follows that $\hat{\mathbb{W}}^{\prime}(t)<0$ for all $t \in\left(0, t_{1}\right]$. Combining with (3.5), we have that for all $t \in\left(0, t_{1}\right]$,

$$
v_{1}^{2}(t)+v_{2}^{2}(t) \leq \frac{\hat{\mathbb{W}}(t)}{\rho_{1}} \leq \frac{\hat{\mathbb{W}}(0)}{\rho_{1}} \leq \frac{\rho_{2}}{\rho_{1}}\left(v_{1}^{2}(0)+v_{2}^{2}(0)\right),
$$

which together with Lemma 3.3 and $u_{K}-u_{0} \in(0,1)$, yields

$$
\begin{aligned}
v_{1}\left(t_{1}\right) & \leq \sqrt{\frac{\rho_{2}}{\rho_{1}}\left(v_{1}^{2}(0)+v_{2}^{2}(0)\right)} \\
& \leq \sqrt{\frac{\rho_{2}\left(c^{2}\left(u_{K}-u_{0}\right)^{2}+K^{2}\left(u_{K}-u_{0}\right)^{2 m}\right)}{\rho_{1} c^{2}}} \\
& \left.\leq\left(u_{K}-u_{0}\right) \sqrt{\frac{\rho_{2}\left(c^{2}+K^{2}\right)}{\rho_{1} c^{2}}}<\sigma<1 \quad \text { (by the definition of } \delta_{1}\right) .
\end{aligned}
$$

Note that $l\left(\bar{u}_{0}, u^{0}\right)\left[\tilde{f}\left(u^{0}\right)-u_{K}\right]>l(a, \bar{u})\left[\widetilde{f}(\bar{u})-u_{K}\right]$ for $a \in\left[\bar{u}_{0}, u_{K}\right)$. Hence $v_{1}>0$ and $v_{2}<0$ on $\left(t_{1}, t_{0}\right]$ by the choice of $c$ and the argument of Lemma 2.3. Therefore $v_{1}(t) \leq v_{1}\left(t_{1}\right)<1$ for all $t \in\left[t_{1}, t_{0}\right]$. To proceed with the proof, we turn to the interval $\left[t_{1}, t_{0}\right]$ and distinguish the following two cases:

(i) $v_{1}(t) \geq 2\left|v_{2}(t)\right| / c$;

(ii) $v_{1}(t)<2\left|v_{2}(t)\right| / c$.

In case (i), we have $c^{2} v_{1}^{m+1}(t)+2 c v_{1}^{m}(t) v_{2}(t) \geq 0$, and hence

$$
\begin{aligned}
\hat{\mathbb{W}}^{\prime}(t) & \leq-\frac{2}{c}[f(u(t))-K]\left(c^{2} v_{1}^{m+1}(t)+2 c v_{1}^{m}(t) v_{2}(t)+5 v_{2}^{2}(t)\right) \\
& \leq-\frac{2}{c}[f(u(t))-K]\left(5 v_{2}^{2}(t)\right) \leq 0 .
\end{aligned}
$$

For case (ii), with the use of $v_{1}<1$ on $\left[t_{0}, t_{1}\right]$, we obtain $2 c v_{1}^{m}(t) v_{2}(t)+4 v_{2}^{2}(t) \geq 0$ for $t \in\left[t_{0}, t_{1}\right]$, and so

$$
\begin{aligned}
\hat{\mathbb{W}}^{\prime}(t) & \leq-\frac{2}{c}[f(u(t))-K]\left(c^{2} v_{1}^{m+1}(t)+2 c v_{1}^{m}(t) v_{2}(t)+5 v_{2}^{2}(t)\right) \\
& \leq-\frac{2}{c}[f(u(t))-K]\left(c^{2} v_{1}^{m+1}(t)+v_{2}^{2}(t)\right) \leq 0 .
\end{aligned}
$$

Therefore we have $\hat{\mathbb{W}}^{\prime}(t) \leq 0$ for all $t \in\left[0, t_{0}\right]$, which establishes the assertion of this claim.

Now by using a similar calculation as in (3.9) and (3.10), we can conclude that

$$
v(t)=v_{1}(t) \leq \sqrt{v_{1}^{2}(t)+v_{2}^{2}(t)} \leq \sqrt{\frac{\rho_{2}\left(c^{2}+K^{2}\right)}{\rho_{1} c^{2}}}\left(u_{K}-u_{0}\right)<\sigma
$$

for all $t \in\left[0, t_{0}\right]$. Furthermore, since $\rho_{2}>\rho_{1}$ and $v(t) \leq u_{K}-u_{0}$ for $t \leq 0$, the above inequality holds for $t \in\left(-\infty, t_{0}\right]$. Using this and noting that $v(t)<1$ on $\left(-\infty, t_{0}\right]$, we 
can obtain from (2.16b) that

$$
\begin{aligned}
u^{\prime}\left(t_{0}\right) & =\frac{1}{d} \int_{-\infty}^{t_{0}} e^{-c\left(t_{0}-s\right) / d} f(u(s)) v^{m}(s) d s \\
& \leq \frac{\widetilde{f}\left(u^{0}\right)}{d} \sqrt{\frac{\rho_{2}\left(c^{2}+K^{2}\right)}{\rho_{1} c^{2}}}\left(u_{K}-u_{0}\right) \int_{-\infty}^{t_{0}} e^{-c\left(t_{0}-s\right) / d} d s \\
& =\frac{\widetilde{f}\left(u^{0}\right)}{c} \sqrt{\frac{\rho_{2}\left(c^{2}+K^{2}\right)}{\rho_{1} c^{2}}}\left(u_{K}-u_{0}\right)<\sigma,
\end{aligned}
$$

which together with (3.11) and $v^{\prime}\left(t_{0}\right)=v_{2}\left(t_{0}\right)<0$, implies that

$$
\left|u\left(t_{0}\right)-\bar{u}\right|<\sigma, \quad\left|u^{\prime}\left(t_{0}\right)\right|<\sigma, \quad v^{\prime}\left(t_{0}\right)<0, \quad \sqrt{v^{2}\left(t_{0}\right)+\left[v^{\prime}\left(t_{0}\right)\right]^{2}}<\sigma .
$$

From this and an application of Lemma 3.2 ( with $u^{0}=\bar{u}$ ), it follows that $u(t) \leq \bar{u}+\delta<u^{0}$ for all $t \geq t_{0}$. Here we have used the fact that the choice of $c$ and the argument of Lemma 2.3 implies that $v(t)>0$ as long as $(u(t), v(t))$ exists. Hence the assertion of this lemma for case (A) is established.

Now we turn to case (B). In this case, we will estimate $u\left(t_{1}\right), v\left(t_{1}\right)$ and $v^{\prime}\left(t_{1}\right)$, and then apply Lemma 3.2 (with $u^{0}=\bar{u}$ and $t_{0}=t_{1}$ ). We first observe that $\hat{\mathbb{W}}^{\prime}(t)<0$ for all $t \in\left(0, t_{1}\right]$ since $v_{1}>0$ and $v_{2}>0$ on $\left[0, t_{1}\right)$. Hence we can use a similar argument as in (3.9) and (3.10) to obtain that

$$
\sqrt{v^{2}(t)+\left[v^{\prime}(t)\right]^{2}} \leq\left(u_{K}-u_{0}\right) \sqrt{\frac{\rho_{2}\left(c^{2}+K^{2}\right)}{\rho_{1} c^{2}}}<\sigma<1 \text { for all } t \in\left[0, t_{1}\right] .
$$

Furthermore, with the argument in (3.12), we see that the estimate for $u^{\prime}\left(t_{0}\right)$ in (3.12) still holds. Next we claim that if $u(t)$ satisfies $u(t) \in\left[\bar{u}, u^{0}\right]$ on $\left[t_{0}, s_{1}\right]$ with $s_{1} \leq t_{1}$, then we have that $u(t) \in[\bar{u}, \bar{u}+\sigma / 2)$ for $t \in\left[t_{0}, s_{1}\right]$. Note that $\bar{u}+\sigma / 2<u^{0}$. To this end we recall that $M_{1}:=\min \left\{\min _{u \in\left[\bar{u}, u^{0}\right]}(f(u)-K), 1\right\}$ and $\mu=2^{\frac{1-m}{2}} \rho_{2}^{-\frac{m+1}{2}} c M_{1}$. Then we can estimate $\hat{\mathbb{W}}^{\prime}$ on $\left[t_{0}, s_{1}\right]$ as follows:

$$
\begin{aligned}
\hat{\mathbb{W}}^{\prime}(t)= & -\frac{2}{c}[f(u(t))-K]\left(c^{2} v_{1}^{m+1}+2 c v_{1}^{m} v_{2}+\frac{c^{2}}{f(u(t))-K} v_{2}^{2}\right) \\
\leq & -2 c\left([f(u(t))-K] v_{1}^{m+1}+v_{2}^{2}\right) \\
\leq & -2 c M_{1}\left(v_{1}^{m+1}+v_{2}^{m+1}\right) \\
& \left(\text { by the definition of } M_{1} \text { and } v_{2}(t) \in[0,1] \text { for } t \in\left[t_{0}, s_{1}\right]\right) \\
\leq & -2^{\frac{1-m}{2}} c M_{1}\left(v_{1}^{2}+v_{2}^{2}\right)^{\frac{m+1}{2}} \\
& \left(\text { since }(a+b)^{p} \leq 2^{p}\left(a^{p}+b^{p}\right) \text { for all } a, b \geq 0 \text { and } p \geq 1\right) \\
\leq & -\mu \hat{\mathbb{W}}^{\frac{m+1}{2}}(t) \quad(\text { by }(3.5) \text { and the definition of } \mu) .
\end{aligned}
$$

From this it follows that

$$
\hat{\mathbb{W}}(t) \leq\left(\hat{\mathbb{W}} \frac{1-m}{2}\left(t_{0}\right)+\frac{\mu(m-1)}{2}\left(t-t_{0}\right)\right)^{\frac{2}{1-m}}
$$


for all $t \in\left[t_{0}, s_{1}\right]$, which together with (2.17a), the estimate of $u^{\prime}\left(t_{0}\right)$ and the definition of $M$, yields

$$
\begin{aligned}
\left|u\left(s_{1}\right)-u\left(t_{0}\right)\right| \leq & \frac{d}{c}\left|u^{\prime}\left(t_{0}\right)\right|+\frac{1}{c} \int_{t_{0}}^{s_{1}} f(u(s)) v^{m}(s) d s \\
\leq & \frac{d}{c}\left|u^{\prime}\left(t_{0}\right)\right|+\frac{M}{c \sqrt{\rho_{1}^{m}}} \int_{t_{0}}^{s_{1}}\left(\hat{\mathbb{W}} \frac{1-m}{2}\left(t_{0}\right)+\frac{\mu(m-1)}{2}\left(s-t_{0}\right)\right)^{\frac{m}{1-m}} d s \\
& (\text { by (3.5) }) \text { and }(\underline{3.14})) \\
\leq & \frac{d}{c}\left|u^{\prime}\left(t_{0}\right)\right|+\frac{2 M}{c \sqrt{\rho_{1}^{m}} \mu} \sqrt{\hat{\mathbb{W}\left(t_{0}\right)}} \\
\leq & \left.\frac{d}{c}\left|u^{\prime}\left(t_{0}\right)\right|+\frac{2 M \sqrt{\rho_{2}}}{c \sqrt{\rho_{1}^{m}} \mu} \sqrt{v_{1}^{2}\left(t_{0}\right)+v_{2}^{2}\left(t_{0}\right)} \quad \text { by (3.5) }\right) \\
\leq & \left(\frac{d \tilde{f}\left(u^{0}\right)}{c^{2}}+\frac{2 M \sqrt{\rho_{2}}}{c \sqrt{\rho_{1}^{m}} \mu}\right) \sqrt{\frac{\rho_{2}\left(c^{2}+K^{2}\right)}{\rho_{1} c^{2}}}\left(u_{K}-u_{0}\right) \\
& \left(\text { by the estimate of } u^{\prime}\left(t_{0}\right) \text { and (3.13) }\right) \\
< & \frac{1}{2} \sigma .
\end{aligned}
$$

This establishes the assertion of this claim. Together with the fact that $\bar{u}+\sigma / 2<u^{0}$, this gives $\left|u\left(t_{1}\right)-\bar{u}\right|<\sigma / 2$. Now we can use the same argument as in (3.12) to obtain that $u^{\prime}\left(t_{1}\right)<\sigma$. Together with (3.13) this yields that

$$
\left|u\left(t_{1}\right)-\bar{u}\right|<\sigma / 2, \quad\left|u^{\prime}\left(t_{1}\right)\right|<\sigma, \quad v^{\prime}\left(t_{1}\right)=0, \quad \sqrt{v^{2}\left(t_{1}\right)+\left[v^{\prime}\left(t_{1}\right)\right]^{2}}<\sigma .
$$

From this and an application of Lemma 3.2 (with $u^{0}=\bar{u}$ and $t_{0}=t_{1}$ ), it follows that $u(t) \leq \bar{u}+\delta<u^{0}$ for all $t \geq t_{1}$. Here we have used the fact that the choice of $c$ and the argument of Lemma 2.3 imply that $v(t)>0$ as long as $(u(t), v(t))$ exists. Hence the assertion of this lemma for case (B) is established, thereby completing the proof of this lemma.

REMARK 3.5. The referee suggests that the application of the Liapunov functions used in the proofs of Lemma 3.2 and Lemma 3.4 can be replaced by a direct argument with the use of the variation of constants formula.

3.2. Proof of Theorem 1.1. With the aid of the lemmas in the last subsection, we are now in a position to establish Theorem 1.1. Indeed, we will modify the shooting argument in Huang [17] to prove Theorem 1.1.

Fix $u^{0}>u_{K}$. Let $\left(u\left(t ; u_{0}, c\right), v\left(t ; u_{0}, c\right)\right)$ denote the unique solution of (2.1) subject to the boundary condition $\lim _{t \rightarrow-\infty}\left(u\left(t ; u_{0}, c\right), u^{\prime}\left(t ; u_{0}, c\right), v\left(t ; u_{0}, c\right), v^{\prime}\left(t ; u_{0}, c\right)\right)=$ $\left(u_{0}, 0,0,0\right)$ with $u_{0} \in\left(0, u_{K}\right)$ and $u\left(0 ; u_{0}, c\right)=u_{K}$, and let $\left(-\infty, T_{u_{0}, c}\right)$ be the corresponding maximal interval on which $\left(u\left(t ; u_{0}, c\right), v\left(t ; u_{0}, c\right)\right)$ exists and $v\left(t ; u_{0}, c\right)$ is positive. Also set $\omega\left(u_{0}, c\right):=\lim _{t \rightarrow T_{u_{0}, c}^{-}} u\left(t ; u_{0}, c\right)$. Then by Lemma 3.1 we can choose (and then fix) a positive number $u_{0}^{*} \in\left(0, u_{K}\right)$ and a sufficiently small positive $\epsilon_{0}$ such that for each $u_{0} \in\left[u_{0}^{*}, u_{0}^{*}+\epsilon_{0}\right]$ and each positive $c$ with $c^{2}>4 l\left(u_{0}, u^{0}\right)\left[\widetilde{f}\left(u^{0}\right)-u_{K}\right]$, we have $\omega\left(u_{0}, c\right)>u^{0}$. Recall that $l(a, b)$ is a decreasing function of $a \in\left(0, u_{K}\right)$ and an increasing function of $b \in\left(u_{K},+\infty\right)$. 
Now fix a positive number $c$ with $c^{2}>4 l\left(u_{0}^{*}, u^{0}\right)\left[\widetilde{f}\left(u^{0}\right)-u_{K}\right]$. We then claim that there is a traveling wave solution of (1.1) with wave speed $c$ connecting $\left(u_{0}, 0\right)$ and $\left(u^{0}, 0\right)$ for some $u_{0} \in\left(0, u_{K}\right)$. Note that if $(u, v)$ is a traveling wave solution of (1.1) connecting $\left(u_{0}, 0\right)$ and $\left(u^{0}, 0\right)$, then by the monotonicity of $u(t)$, we have $u(t) \in\left(u_{0}, u^{0}\right)$ for all $t \in \mathbb{R}$. Therefore redefining the value $f(u)$ for $u>u^{0}$ will not affect the wave solution $(u, v)$. Hence we can redefine the value $f(u)$ to be $f\left(u^{0}\right)$ for each $u>u^{0}$, and so assumption (A4) is automatically satisfied. To continue the proof, we consider the set

$$
\mathcal{A}_{u^{0}}:=\left\{u_{0} \in\left(0, u_{K}\right): \omega\left(u_{0}, c\right)<u^{0}\right\} .
$$

Note that $l\left(u_{0}^{*}, u^{0}\right)>l\left(a, u^{0}\right)$ for $a \in\left(u_{0}^{*}, u_{K}\right)$. Hence by Lemma 3.4 we have $\left[\hat{u}_{0}, u_{K}\right) \subseteq$ $\mathcal{A}_{u^{0}}$ for some positive $\hat{u}_{0}$. On the other hand, by the choice of $u_{0}^{*}$ and $\epsilon_{0}$, and the fact that $l\left(u_{0}^{*}, u^{0}\right)>l\left(u_{0}, u^{0}\right)$ for $u_{0} \in\left(u_{0}^{*}, u_{0}^{*}+\epsilon_{0}\right]$, we can obtain that $u_{0} \notin \mathcal{A}_{u^{0}}$ for each $u_{0} \in\left[u_{0}^{*}, u_{0}^{*}+\epsilon_{0}\right]$. Hence the quantity

$$
\alpha_{u^{0}}:=\inf \mathcal{A}_{u^{0}}
$$

is well-defined and lies in the interval $\left(u_{0}^{*}, u_{K}\right)$. Now we claim that $\omega\left(\alpha_{u^{0}}, c\right)=u^{0}$. Suppose not. Then one of the following cases holds:

(i) $\omega\left(\alpha_{u^{0}}, c\right)>u^{0}$;

(ii) $\omega\left(\alpha_{u^{0}}, c\right)<u^{0}$.

We first deal with case (i).

From the proof in Lemma 2.1 and the standard theory of continuous dependence on the initial value for differential equations on a finite time interval, we can conclude that for each $u_{0} \in\left(0, u_{K}\right)$ and each finite number $S<T_{u_{0}, c}$, there exists a sufficiently small $\delta>0$ such that for each $a \in\left[u_{0}-\delta, u_{0}+\delta\right]$, the solution $(u(t ; a, c), v(t ; a, c))$ exists on $(-\infty, S]$ and depends continuously on $a \in\left[u_{0}-\delta, u_{0}+\delta\right]$. Therefore if $\tilde{u}_{0}>\alpha_{u^{0}}$ is sufficiently close to $\alpha_{u^{0}}$, then we can use this continuity property and the monotonicity of $u\left(t ; \tilde{u}_{0}, c\right)$ to obtain that $\omega\left(\tilde{u}_{0}, c\right)>u^{0}$. This implies that inf $\mathcal{A}_{u^{0}}>\alpha_{u^{0}}$, a contradiction. For case (ii), we first note that $l\left(\alpha_{u^{0}}, \omega\left(\alpha_{u^{0}}, c\right)\right)<l\left(u_{0}^{*}, u^{0}\right)$ by the property of $l(a, b)$. Together with Lemma 2.3, we see that $\left(u\left(t ; \alpha_{u^{0}}, c\right), v\left(t ; \alpha_{u^{0}}, c\right)\right)$ is globally defined. Then we can use the above continuity property in case (i), Lemma 3.2 and Lemma 2.3 to deduce that if $\tilde{u}_{0}<\alpha_{u^{0}}$ is sufficiently close to $\alpha_{u^{0}}$, then we have $\omega\left(\tilde{u}_{0}, c\right)<u^{0}$, and so $\inf \mathcal{A}_{u^{0}}<\alpha_{u^{0}}$. This is a contradiction again. Therefore we have $\omega\left(\alpha_{u^{0}}, c\right)=u^{0}$.

Finally, since $c^{2}>4 l\left(u_{0}^{*}, u^{0}\right)\left[\widetilde{f}\left(u^{0}\right)-u_{K}\right]>4 l\left(\alpha_{u^{0}}, u^{0}\right)\left[\widetilde{f}\left(u^{0}\right)-u_{K}\right]$, it follows from Lemma 2.3 that $\left(u\left(t ; \alpha_{u^{0}}, c\right), v\left(t ; \alpha_{u^{0}}, c\right)\right)$ is globally defined. Hence $\left(u\left(t ; \alpha_{u^{0}}, c\right)\right.$, $\left.v\left(t ; \alpha_{u^{0}}, c\right)\right)$ is a traveling wave solution of (1.1) connecting $\left(\alpha_{u^{0}}, 0\right)$ and $\left(u^{0}, 0\right)$, thereby completing the proof of this theorem.

Acknowledgements. The author would like to thank Professor Masayasu Mimura for drawing the author's attention to this model. The author also would like to thank the referees for their suggestions.

\section{REFERENCES}

[1] Ai, S. and Huang, W. (2005) Traveling waves for a reaction-diffusion system in population dynamics and epidemiology. Proc. Roy. Soc. Edinburgh Sect. A 135A, 663-675. MR2173333 (2006e:35188) 
[2] Ai, S. and Huang, W. (2007) Traveling wavefronts in combustion and chemical reaction models. Proc. Roy. Soc. Edinburgh Sect. A 137A, 671-700. MR 2345776 (2008g:35106)

[3] Bailey, N. T. J. (1975) The mathematical theory of infectious diseases and its applications. 2nd edition, Hafner Press. MR0452809 (56:11084)

[4] Ballyk, M., Dung, L., Jones, D. A. and Smith, H. L. (1999) Effects of random motility on microbial growth and competition in a flow reactor. SIAM J. Appl. Math. 59, 573-596. MR1654407 (2001a:92027)

[5] Billingham, J. and Needham, D. J. (1991a) The development of travelling waves in quadratic and cubic autocatalysis with unequal diffusion rates. I. Permanent form travelling waves. Philos. Trans. R. Soc. Ser. A 334, 1-24. MR1155096 (92m:80020)

[6] Billingham, J. and Needham, D. J. (1991b) The development of travelling waves in quadratic and cubic autocatalysis with unequal diffusion rates. II. An initial value problem with immobilised or nearly immobilised autocatalyst. Philos. Trans. R. Soc. Ser. A 336, 497-539. MR1133118 (93a:80012)

[7] Carr, J. (1981) Applications of centre manifold theory. Springer-Verlag, New York. MR635782 (83g:34039)

[8] Chen, X. and Qi, Y. (2007) Sharp estimates on minimum traveling wave speed of reaction diffusion systems modelling autocatalysis. SIAM J. Math. Anal. 39, 437-448. MR2338414 (2008h:34083)

[9] Fisher, R. A. (1937) The wave of advance of advantageous genes. Ann. Eugenics 7, 353-369.

[10] Gowland R. J. and Stedman, G. (1983) A novel moving boundary reaction involving hydroxylamine and nitric acid. J. Chem. Soc., Chem. Commun. 10, 1038-1039.

[11] Gray, P. (1988) Instabilities and oscillations in chemical reactions in closed and open systems. Proc. R. Soc. A 415, 1-34.

[12] Guo, J.-S. and Tsai, J.-C. (2009) Traveling waves of two-component reaction-diffusion systems arising from higher order autocatalytic models. Quart. Appl. Math. 67, 559-578. MR 2547640

[13] Hodgkin, A. L. and Huxley, A. F. (1952) A quantitative description of membrane current and its application to conduction and excitation in nerve. J. Physiol. (London) 117, 500-544.

[14] Hosono, Y. and Ilyas, B. (1994) Existence of traveling waves with any positive speed for a diffusive epidemic model. Nonlin. World 1, 277-290. MR.1303097 (95k:92021)

[15] Hosono, Y. and Ilyas, B. (1995) Travelling waves for a simple diffusive epidemic model. Math. Models Meth. Appl. Sci. 5, 935-966. MR1359215 (96j:35248)

[16] Hosono, Y. (2007) Phase plane analysis of travelling waves for higher order autocatalytic reactiondiffusion systems. Discrete Contin. Dyn. Syst. Ser. B 8, 115-125. MR2300326 (2008c:35148)

[17] Huang, W. (2004) Travelling waves for a biological reaction-diffusion model. J. Dynam. Diff. Eqns. 16, 745-765. MR 2109164 (2005g:35175)

[18] Huang, W. (2006) Uniqueness of traveling wave solutions for a biological reaction-diffusion equation. J. Math. Anal. Appl. 316, 42-59. MR2201748 (2006k:35149)

[19] Källén, A. (1984) Thresholds and traveling waves in an epidemic model for rabies. Nonlinear Anal. TMA 8, 851-856. MR753763 (86h:92042)

[20] Kay, A. L., Needham, D. J. and Leach, J. A. (2003) Travelling waves for a coupled, singular reactiondiffusion system arising from a model of fractional order autocatalysis with decay: I. Permanent form travelling waves. Nonlinearity 16, 735-770. MR1959108 (2004c:35215)

[21] Kennedy, C. R. and Aris, R. (1980) Travelling waves in a simple population model involving growth and death. Bull. Math. Biol. 42, 397-429. MR661329 (84f:92041)

[22] Kermack, W. O. and McKendric, A. G. (1927) Contribution to the mathematical theory of epidemic. Proc. R. Soc. A 115, 700-721.

[23] Kolmogorov, A. N., Petrovsky, I. G. and Piskunov, N. S. (1937) Étude de l'équation de la diffusion avec croissance de la quantité de matière et son application à un problème biologique. Bull. Université d'État à Moscou, Ser. Int., Sect. A. 1, 1-25.

[24] Marion, M. (1985) Qualitative properties of a nonlinear system for laminar flames without ignition temperature. Nonlin. Analysis 9, 1269-1292. MR813658 (87j:80016)

[25] Merkin, J. H. and Needham, D. J. (1989) Propagation reaction-diffusion waves in a simple isothermal quadratic chemical system. J. Eng. Math. 23, 343-356. MR1029938 (90k:80024)

[26] Merkin, J. H. and Needham, D. J. (1990) The development of travelling waves in a simple isothermal chemical system. II. Cubic autocatalysis with quadratic and linear decay. Proc. R. Soc. A 430, 315345. MR1068302 (91i:80008) 
[27] Merkin, J. H. and Needham, D. J. (1991) The development of travelling waves in a simple isothermal chemical system. IV. Quadratic autocatalysis with quadratic decay. Proc. R. Soc. A 434, 531-554.

[28] Merkin, J. H. and Needham, D. J. (1993) Reaction-diffusion waves in an isothermal chemical system with general orders of autocatalysis and spatial dimension. J. Appl. Math. Phys. (ZAMP) 44, 707721. MR.1239888 (94f:35067)

[29] Needham, D. J. and Merkin, J. H. (1991) The development of travelling waves in a simple isothermal chemical system with general orders of autocatalysis and decay. Philos. Trans. Roy. Soc. London Ser. A 337, 261-274. MR.1143726 (93a:80013)

[30] Qi, Y. (2007) The development of traveling waves in cubic auto-catalysis with different rates of diffusion. Physica D. 226, 129-135. MR2296235 (2007k:35270)

[31] Saul, A. and Showalter, K. (1984) Propagating reaction-diffusion fronts. In Oscillations and Traveling waves in chemical systems (ed. R. J. Field and M. Burger), Wiley, New York.

[32] Smith, H. L. and Zhao., X. Q. (2004) Travelling waves in a bio-reactor model. Nonlin. Analysis Real World Applic. 5, 895-909. MR.2085700 (2005g:35161)

[33] Voronkov, V. G. and Semenov, N. N. (1939) Zh. Fiz. Khim. 13, 1695.

[34] Zaikin, A. N. and Zhabotinskii, A. M. (1970) Concentration wave propagation in two-dimensional liquid-phase self-oscillating system. Nature 225, 535-537. 